\title{
Élaboration du rendement du blé d'hiver en conditions de déficit hydrique. II. Mise au point et test d'un modèle de simulation de la culture de blé d'hiver en conditions d'alimentation hydrique et azotée variées : Epicphase-Blé
}

\author{
P Debaeke *, M Cabelguenne, ML Casals, J Puech
}

Station d'agronomie, Inra, BP 27, F-31326 Castanet-Tolosan cedex, France

(Reçu le 30 mai 1995 ; accepté le 8 janvier 1996)

\begin{abstract}
Résumé - Le modèle Epic (Williams et al, 1989) est couramment utilisé pour simuler le fonctionnement du blé dans une rotation culturale bien qu'il surestime le rendement en présence de forte contrainte hydrique. Une version remaniée (Epicphase E) a été développée à l'Inra pour améliorer la performance du modèle en conditions d'eau limitée. Les affinements introduits comprennent notamment : i) une meilleure description du profil d'extraction de l'eau ; ii) une réponse de l'indice de récolte aux stress hydrique et azoté par phase du cycle. Cet article décrit ces améliorations et propose un étalonnage du modèle pour le blé d'hiver. La recherche des paramètres s'effectue à partir des données de la littérature et de dispositifs expérimentaux (lysimètres, essai pluriannuel au champ). L'étalonnage concerne le fonctionnement du blé en l'absence puis en présence de contrainte hydrique (accumulation et répartition de la biomasse, mise en place des feuilles et des racines, prélèvement d'eau). Le test du modèle est réalisé au champ à Auzeville (Haute-Garonne, France) au cours de deux années (1989 et 1990) présentant des conditions de sécheresse marquées et des conditions d'alimentation azotée bien différenciées. Si l'on tient compte de la variabilité des conditions testées, on peut conclure à la bonne prévision par le modèle de la consommation en eau, de la biomasse aérienne, du rendement et de l'azote absorbé, avec des écarts relatifs moyens de 8 à $18 \%$ selon les variables et les années. La durée semis-épiaison est prévue avec un écart moyen de 59 degrés $x$ jours. L'utilisation du modèle Epicphase pour affiner la conduite de la culture du blé en conditions hydriques plus ou moins limitantes paraît ainsi envisageable.
\end{abstract}

blé d'hiver / modèle de simulation / rendement / contrainte hydrique / conduite de la culture

Summary - Yield build-up in winter wheat under soil water deficit. II. Development and testing of a simulation model for wheat under various water and nitrogen regimes: EPICPHASE-Wheat. The EPIC model (Williams et al, 1989) is commonly used to simulate the wheat crop in a crop rotation, though it overestimates grain yield in dry conditions. A new version of the model (EPICPHASE) has been developed by INRA in Toulouse to improve the model performance in drought-prone environments. The refinements introduced were: 1) a better description of the soil water extraction pattern; 2) a response of harvest index to water and nitrogen stress as a function of phenological phase. This study describes this new version and proposes the corresponding calibration for winter soft wheat. Literature and various experiments (lysimeters, pluri-annual field testing) were used to determine the optimal set of parameters. 
Functions describing wheat growth without limiting factors under water constraint were calibrated (ie, biomass accumulation and partitioning, leaf area and root depth, water uptake). The model was tested in Auzeville (Haute-Garonne, SW France) during two successive dry years (1989 and 1990) differing in their drought pattern, and on a range of fields differing in water and nitrogen supply. Referring to the extreme variability of the test situations, the predictions of the model were satisfactory for water budget, biomass, yield and nitrogen uptake, with mean relative errors of 8-18\%, depending on variable and year. The sowing-heading duration was predicted with a root mean squared error of 59 degree.days. The EPICPHASE-Wheat model should be quite valuable for assessing the impact of different management schemes in rainfed or irrigated wheat crops and for defining optimum strategies.

winter wheat / simulation model / yield / water deficit / crop management

\section{INTRODUCTION}

Dans les régions où l'eau est un facteur limitant de la production du blé, les besoins en outils d'aide à la décision sont de deux types : i) la conception d'itinéraires techniques optimisant l'utilisation de la ressource hydrique (choix de la date de semis, de la variété, de la densité de peuplement, de la fumure azotée), ii) l'aide au raisonnement de l'irrigation de complément du blé (besoins en eau, stades d'apports). En effet, en raison de la faible valorisation de l'irrigation et de la variabilité d'apparition des déficits hydriques entre années, le blé d'hiver nécessite la mise en œuvre d'outils de pilotage de l'irrigation (Bouthier et Bonnefoy, 1993). Plusieurs auteurs (Fischer, 1985 ; Stapper et Harris, 1989; Singels, 1992) ont montré comment ces deux types de questions pouvaient être résolues par l'utilisation de modèles de simulation de la culture de blé.

La modélisation du rendement (ou de la biomasse aérienne) est assurée de deux manières :

- directement, par des liaisons statistiques entre la transpiration, le rayonnement intercepté ou diverses variables agroclimatiques (Rasmussen et Hanks, 1978 ; Dagneaud et Tranchefort, 1985 ; Farshi et al, 1987);

- à l'issue d'une simulation dynamique des processus de croissance et de développement, aboutissant au rendement par l'élaboration des composantes successives de la production (Whisler et al, 1986). Le niveau de description et d'explication des processus biophysiques est souvent assez fin mais la validation au champ est entravée par la nécessité d'estimer un grand nombre de paramètres.

Ces modèles mécanistes sont devenus plus opérationnels pour l'agronome dès lors qu'ils ont intégré l'effet des contraintes hydriques et azotées. Des exemples de ces modèles complexes, synthèses achevées des connaissances agro- physiologiques, sont rapportés pour le blé par Maas et Arkin (1980), Weir et al (1984), Baker et al (1985), O'Leary et al (1985), Ritchie et Otter (1985), van Keulen et Seligman (1987), Stockle et Campbell (1989), Hansen et al (1991) et Porter (1993). Ces modèles diffèrent notablement par la description des processus mais aussi par les efforts d'évaluation entrepris à ce jour. Les effets de fortes contraintes hydriques et azotées en interaction (Brisson et Delécolle, 1993) tout comme les transferts d'assimilats entre organes (Russel et Wilson, 1994) restent encore imparfaitement décrits.

Les modèles simulant le fonctionnement d'un peuplement de blé placé dans une succession culturale sont moins nombreux (Littleboy et al, 1989 ; Stockle et al, 1994). Parmi ceux-ci, le modèle Epic (Erosion productivity impact calculator), développé par Williams et al (1984, 1989), a été utilisé dans le sud-ouest de la France pour simuler la réponse des principales grandes cultures placées en rotations et diversement intensifiées (Cabelguenne et al, 1988, 1990). Cependant, lorsque le déficit hydrique est prononcé, Epic surestime à la fois la biomasse totale et l'indice de récolte, ce qui conduit à de fortes erreurs de prédiction sur le rendement. C'est pourquoi, des affinements du modèle ont été proposés pour améliorer la simulation de l'extraction d'eau et la réponse de l'indice de récolte à la contrainte hydrique ; un découpage du cycle en phases phénologiques a permis de moduler les effets du stress au cours du cycle. L'intégration à l'échelle de la succession des cultures de ce cadre général mis au point sur maïs et tournesol (Quinones, 1989 ; Texier, 1992) a conduit à la version Epicphase (Cabelguenne et al, 1994) qu'il convient à présent de valider pour les principales grandes cultures à graines.

C'est pourquoi, après avoir rappelé la structure du modèle Epicphase, nous procéderons dans cet article à l'étalonnage et à l'évaluation de ce modèle pour le blé d'hiver en conditions d'alimentation hydrique et azotée différenciées. 


\section{MATÉRIEL ET MÉTHODES}

\section{Rappel de la structure générale du modèle Epic}

Epic simule la croissance journalière d'une culture en fonction de l'évolution des stocks d'eau et d'éléments minéraux du sol ( $\mathrm{N}$ et $\mathrm{P}$ ). À la récolte, la biomasse aérienne est convertie en rendement-grain au moyen d'un indice de récolte. Les entrées principales du modèle décrivent le climat (radiation, pluviométrie, températures maximales et minimales, humidité relative de l'air, vitesse du vent), le sol (texture, profondeur, humidités à la capacité au champ et au point de flétrissement, azote organique, azote minéral, carbone) et les itinéraires techniques (dates de semis et de récolte, culture et variété, fertilisation NP, irrigation). Les effets dépressifs des pathogènes, adventices et ravageurs sur la biomasse ne sont pas simulés.

L'annexe I résume les principales équations d'Epic qui régissent le fonctionnement d'une culture en l'absence de stress hydrique ou azoté : accumulation et répartition de la biomasse entre organes, cinétique et mise en place des capteurs (feuilles, racines) et prélèvement d'eau et d'azote. L'annexe II résume les équations de réponse de l'indice foliaire et de la biomasse totale aux stress hydriques et azotés, selon la loi du facteur limitant principal (Eq 13 et 16). Alors que la réponse de la biomasse est proportionnelle au stress hydrique, la croissance est nulle pour un stress azoté de 0,5 (Eq 14 et 15). De même, un stress azoté ou hydrique limite l'accroissement d'indice foliaire (expansion et élongation confondues) en phase d'établissement (Eq 17).

\section{Améliorations apportées dans la version Epicphase}

L'analyse critique du fonctionnement du modèle Epic en conditions d'eau limitée (Quinones, 1989) a conduit à plusieurs améliorations qui ont été rassemblées dans la version Epicphase et que l'on résume ici.

\section{Découpage du cycle en phases}

Epicphase comprend une partition du cycle en quatre phases de développement, définies par les sommes de températures utiles. Ceci permet de distinguer des phases de sensibilité différente au stress hydrique et d'envisager une modulation de la valeur de RUE (Eq 1) par phase. Pour le blé, nous avons retenu le découpage suivant : levée-début montaison (D1), début montaison-gonflement (D2), gonflement-grain laiteux (D3), grain laiteux-maturité (D4). La durée relative des phases dépend de la variété et de la date de semis; or le modèle ne reconnaît pas d'effet vernalisant ou photopériodique. En revanche, en culture d'hiver, le modèle réinitialise les sommes de température le 15 janvier afin de corriger en partie l'effet de la date de semis. Le découpage des phases se calcule ainsi sur la durée 15 janvier-maturité, moins variable selon la date de semis pour un même cultivar.

\section{Réponse de l'indice de récolte aux stress hydriques et azotés}

Dans un précédent article (Debaeke et al, 1996), nous avons détaillé le modèle de réponse de l'indice de récolte $(\mathrm{IR})$ du blé au stress hydrique pour chacune des quatre phases précédentes. La variation de l'indice de récolte (par rapport à $\mid R_{0}$ ) est mise en relation avec le nombre de jours de stress hydrique subis par le blé pendant la phase considérée, la pente $\left(\mathrm{PSH}_{j}\right)$ exprimant la sensibilité de la phase. Seuls les stress hydriques inférieurs à LIMSTR $(0,8$ pour le blé) sont retenus. Cette approche spécifique remplace celle d'Epic qui considère qu'à partir de $55 \%$ du cycle une valeur d'ETR/ETM de 0,5 abaisse IR de $10 \%$ quelle que soit la culture. La même démarche est adoptée pour traduire l'effet d'un stress azoté, alors qu'Epic ne prévoit pas d'effet du manque d'azote sur IR. En accord avec Donald et Hamblin (1976), les effets des stress hydrique et azoté sur l'indice de récolte sont cumulés, alors que la loi du facteur limitant est appliqué pour la biomasse totale. Ainsi, à maturité, on calcule IR de la façon suivante:

$$
\text { (18) IR }=\mathrm{IR}_{0}+\sum_{i=1}^{4}\left(\mathrm{PSH}_{i}{ }^{*} \mathrm{NJSH}_{i}\right)+\sum_{i=1}^{4}\left(\mathrm{PSN}_{i}{ }^{\star} \mathrm{NJSN}_{i}\right)
$$

pour $\mathrm{NJSH}_{i}=\mathrm{NJ}_{i}{ }^{*}\left(1-\mathrm{ETR}_{i} / \mathrm{ETM}_{i}\right)$ et $\mathrm{NJSN}_{i}=\mathrm{NJ}_{i}{ }^{*}$ $\left(1-\mathrm{SN}_{i}\right)$

$\mathrm{IR}_{0}=$ indice de récolte d'une culture bien alimentée en eau et en azote ; $\mathrm{PSH}_{i}$ : paramètre $(>0$ ou $<0$ ) exprimant la sensibilité de la phase i au stress hydrique ; $\mathrm{PSN}_{i}$ : paramètre $(>0$ ou $<0$ ) exprimant la sensibilité de la phase $i$ au stress azoté ; $\mathrm{NJSH}_{i}$ : nombre de jours de stress hydrique de la phase $i ; \mathrm{NJSN}_{i}$ : nombre de jours de stress azotés de la phase $i ; \mathrm{NJ}_{i}$ : durée de la phase $i$ (jours)

\section{Extraction de l'eau du sol}

Lorsque le potentiel hydrique du sol ne limite pas la transpiration, le prélèvement d'eau journalier ne dépend que de la profondeur considérée et de la forme d'enracinement de la culture (Eq 9). Epic considère que l'extraction de l'eau est identique pour toutes les cultures, avec un enracinement de type conique prélevant $60 \%$ de l'eau consommée sur le tiers supérieur du système racinaire (soit $\alpha=10$ ). Ce modèle unique n'est pas valable pour toutes les cultures, tous les sols ou toutes les conduites culturales (Maertens et Marty, 1972 ; Quinones, 1989). Aussi Epicphase admet un choix d' $\alpha$ modulable selon les cultures et les conditions d'exploration racinaire. La valeur d'ETM $(E q$ 10) est déduite de l'évapotranspiration de référence de la culture (ETP , formule de Penman-Monteith) et de la 
valeur d'indice foliaire $\left(\mathrm{IF}_{j}\right)$, selon la méthode proposée par Ritchie (1972). Epicphase considère cependant que le besoin hydrique peut être augmenté audelà de l'ETP au cours de certaines phases du cycle, variables selon les cultures, selon l'équation :

$$
\text { (19) } \begin{aligned}
\mathrm{ETM}_{j} & =\left(1+\mathrm{K}_{m x}\right)^{*} \mathrm{ETP}_{j} \\
0 & <\mathrm{K}_{m x}<1
\end{aligned}
$$

En cas de fourniture en eau insuffisante pour un horizon donné, Epic prévoit une compensation par les autres horizons ( $\mathrm{Eq} 11)$. Une compensation complète $(p=1)$ a pour conséquence de retarder l'apparition des stress hydriques puis de les faire agir de manière brutale et irréversible, une fois les réserves épuisées ou de les faire agir à des phases de sensibilité décalées (Quinones, 1989). Epicphase permet de moduler le paramètre $p$ selon les sols et les cultures (Eq 11).

Lorsque la transpiration est limitée par le potentiel hydrique du sol, la culture fonctionne à l'ETR (Eq 12). Le prélèvement s'effectue pour l'essentiel sur la gamme d'eau utile définie par le point de flétrissement (HPF) et la capacité au champ (HCC) ; cependant, de fortes dessiccations, en deçà de HPF, sont fréquemment observées au champ pour les principales cultures (Maertens et Cabelguenne, 1974). Epic permet ce dépassement de manière uniforme jusqu'à $50 \mathrm{~cm}$ de profondeur alors qu'Epicphase autorise une extraction en deçà du point de flétrissement plus profonde $(60 \mathrm{~cm})$ mais décroissante avec la profondeur. La dessiccation maximale retenue pour le blé est comprise entre $5 \%$ de HPF $(0-10 \mathrm{~cm})$ et $50 \%$ de HPF $(40-60$ $\mathrm{cm}$ ) mais pourrait être modulée selon les types de sol.

Selon Epic, le prélèvement en eau de l'horizon $h$ n'est affecté qu'à partir d'un taux de remplissage de la réserve utile inférieur ou égal à $25 \%$, quels que soient les sols et les cultures. Or cette limite de réserve facilement utilisable, souvent évoquée pour le blé (Russell et Wilson, 1994), ne peut être considérée comme universelle (Puech, 1972). Aussi, le paramètre $t$ a-t-il été introduit dans Epicphase (Eq 12), afin de permettre la modulation de cette limite selon les conditions ; $t$ permet ainsi d'exprimer la cessibilité réduite de l'eau en conditions de faible humidité du sol ou de texture argileuse. Puisque le transfert d'azote vers les racines s'exerce principalement par le flux de transpiration, l'alimentation azotée sera pénalisée en conséquence par une faible consommation hydrique de la culture (Williams et al, 1984).

\section{Effet du stress hydrique sur la vitesse de sénescence foliaire}

Un stress hydrique marqué accélère la sénescence des feuilles et réduit la durée de surface foliaire après floraison. Aussi Texier (1992) a modifié le paramètre de sénescence foliaire (dIF) afin de permettre une accélération significative de la régression de surface foliaire dès $50 \%$ du cycle (soit 8 jours avant floraison) dans le cas de stress hydriques marqués ; ainsi la valeur corrigée de diF tient compte de la moyenne des stress hydriques pris sur 5 jours consécutifs $\left(\mathrm{STR}_{5 j \mathrm{rs}}\right)$ et d'un paramètre d'accélération de la sénescence (acc) :

$$
\text { (20) } d I F_{c o r}=d I F+a c c^{\star} d I F^{\star}\left(1-S T R_{5 j r s}\right)
$$

Ces affinements s'ajoutent aux équations des annexes I et II ou les modulent.

\section{Données expérimentales utilisées pour l'étalonnage et l'évaluation d'Epicphase}

Les données expérimentales proviennent de deux dispositifs situés à Auzeville (Haute-Garonne, France) sur le domaine de l'Inra.

- Un essai pluriannuel de plein champ (1984-1993), où I'on compare, en sol limono-argileux profond, le fonctionnement de rotations irriguées ou non, conduites selon deux ou trois niveaux d'intrants (Debaeke et Hilaire, 1991). Chaque année, 25 parcelles de $550 \mathrm{~m}^{2}$ sont conduites en blé d'hiver. Elles varient par la nature du précédent cultural et par la fertilisation azotée (apports raisonnés $(N)$ selon des objectifs de rendement de 55,70 et 85 q.ha-1). Une zone témoin ne recevant pas d'engrais azoté sur blé (ON) est installée sur chaque parcelle. Un suivi neutronique des profils hydriques jusqu'à une profondeur de $1,70 \mathrm{~m}$ est pratiqué chaque année sur un échantillon de parcelles de blé. Les principaux stades phénologiques (levée, stade "épi $1 \mathrm{~cm}$ ", épiaison, floraison, stade laiteux, maturité) sont relevés. Des prélèvements de biomasse aérienne, des mesures d'indice foliaire par planimétrie et des déterminations d'azote absorbé (méthode Kjeldahl) ont été effectués sur $5 \mathrm{x}$ $0,18 \mathrm{~m}^{2}$ du tallage à la maturité du blé en 1986 (cv Talent) et 1993 (cv Soissons) sur un échantillon de parcelles $\mathrm{N}$ et $\mathrm{ON}$. À maturité, la biomasse aérienne, le rendement, l'indice de récolte et la teneur en azote du grain et de la paille sont estimés à partir de deux prélèvements de $0,5 \mathrm{~m}^{2}$ par traitement.

- Des dispositifs contrôlés composés de grandes cases lysimétriques (cV Top 1982, Vizir 1991, Soissons 1994), de petits lysimètres et d'évapotranspiromètres (cv Top 1982), décrits en détail par ailleurs (Debaeke et al, 1996), qui ont servi à étudier la dynamique de prélèvement de l'eau et la réponse du blé au stress hydrique selon les phases du cycle, la profondeur et la texture du sol.

Parmi les données du dispositif au champ, seules les années sans facteurs limitants majeurs (pas de sécheresse, pas d'attaque de rouille brune) et les conduites intensives (apports d'eau et d'azote élevés, bonne protection phytosanitaire) ont servi à étalonner les paramètres de croissance du blé et la réponse de l'indice de récolte au stress azoté. La démarche suivante a été menée pour étalonner les principales fonctions d'Epicphase : 
- recherche bibliographique des paramètres culturaux difficilement mesurables (efficience de conversion du rayonnement, coefficient d'extinction de la lumière...);

- détermination des paramètres d'enracinement et d'évolution de l'indice foliaire à partir des données du champ (années 1985 et 1986/1993, respectivement) ;

- étalonnage du profil d'extraction d'eau à partir des grandes cases lysimétriques (année 1994), variant par leur profondeur $(0,95$ à $2,25 \mathrm{~m})$ et leur cessibilité en eau (cases argilo-limoneuses ou limoneuses), et pour lesquelles on dispose de traitements où la dessiccation est continue ;

- étalonnage de la réponse de l'indice de récolte à la contrainte hydrique, à l'aide des lysimètres précédents (année 1991) ;

- étalonnage de la réponse de l'indice de récolte au défaut d'alimentation azotée à partir des données du champ 1993.

L'évaluation du modèle Epicphase, pratiquée sur les mêmes dispositifs que précédemment (cases et champ) mais en changeant d'année ou de traitement expérimental, s'est opérée en plusieurs étapes :

- en l'absence de contrainte hydrique, validation de la simulation de la biomasse aérienne au cours du cycle et de la formation de l'indice de récolte (champ 1993, cv Soissons), simulation du prélèvement d'eau à l'ETM (évapotranspiromètres 1982, cv Top);

- évaluation de la consommation hydrique (ETR, mm) pour des situations variées de disponibilité en eau (cases 1982, cv Top) ;

- test au champ lors de deux années sèches (cv Vizir 1989 et 1990) : évaluation des variables rendement (RDT, tha ${ }^{-1}$ ), biomasse aérienne (MST, t.ha-1), indice de récolte (IR), eau consommée (ETR, $\mathrm{mm}$ ) et azote absorbé (NABS, $\mathrm{kg} \mathrm{ha}^{-1}$ ). Les écarts entre valeurs simulées et mesurées ont été comparés aux écarts obtenus avec la version d'EPIC antérieure. L'année 1989 se caractérise par un déficit climatique prononcé dès l'épiaison (tableau I) et des conditions de remplissage du grain très échaudantes (12 jours où la température maximale a dépassé $28^{\circ} \mathrm{C}$ ) ; l'année 1990 se caractérise par une sécheresse continue dès le stade "épi $1 \mathrm{~cm}$ ", accentuée par l'absence de recomblement des réserves du sol en hiver. En 1990, on dispose de parcelles de blé irriguées $(75 \mathrm{~mm}$ ) ou non, alors qu'en 1989 il n'y a pas eu d'irrigation de complément ;

- test des variables RDT, MST, IR et ETR en lysimètres (cv Vizir 1991, cv Soissons 1994), dans les situations de déssèchement continu (Debaeke et al, 1996) qui n'ont pas servi à l'étalonnage. On cherche ici à éprouver la validité du modèle en condition de sécheresse prolongée où des processus d'adaptation sont possibles.

La performance d'un modèle peut être évaluée par plusieurs indicateurs statistiques (Willmott, 1982) ; parmi ceux-ci, nous retiendrons RMSE, racine carrée de la moyenne de la somme des carrés des écarts entre observation (OBS) et simulation (SIM), et ERM = RMSE/moyOBS, que nous appellerons écart relatif moyen.

\section{Recherche des paramètres d'Epicphase pour le blé d'hiver}

Les paramètres d'étalonnage du modèle Epicphaseblé sont rassemblés au tableau II.

Tableau I. Données climatiques mensuelles sur le cycle du blé d'hiver en région toulousaine.

Nov $\frac{}{\text { Déc }} \underset{\text { Jan }}{\text { Fév }} \frac{\text { Mois }}{\text { Mar }} \frac{}{\text { Avr }}-\frac{}{\text { Mai }} \frac{}{\text { Juin }} \frac{}{\text { Juil }}$

Moyenne 45 ans (1949-1993)

$\begin{array}{lrrrrrrrrr}\text { Pluie }(\mathrm{mm}) & 51 & 64 & 68 & 60 & 73 & 61 & 94 & 71 & 48 \\ \text { ETP }(\mathrm{mm}) & 29 & 19 & 22 & 40 & 76 & 103 & 129 & 147 & 172 \\ \text { Pluie-ETP }(\mathrm{mm}) & 22 & 45 & 46 & 20 & -3 & -42 & -35 & -76 & -124 \\ & & & & & & & & & \\ 988-1989 & 50 & 5 & 23 & 58 & 42 & 113 & 9 & 9 & 6 \\ \text { Pluie }(\mathrm{mm}) & 31 & 27 & 17 & 34 & 76 & 75 & 148 & 152 & 179 \\ \text { ETP }(\mathrm{mm}) & 19 & -22 & 6 & 24 & -34 & 38 & -139 & -143 & -173 \\ \text { Pluie-ETP }(\mathrm{mm}) & & & & & & & & & \end{array}$

$1989-1990$

Pluie $(\mathrm{mm})$

$\operatorname{ETP}(\mathrm{mm})$

Pluie-ETP $(\mathrm{mm})$

54
16
38

$\begin{array}{rrr}56 & 21 \\ 8 & 14 \\ 48 & 7\end{array}$

78
47
31

5
82
-77

$\begin{array}{rrr}66 & 86 & 38 \\ 78 & 134 & 114 \\ -12 & -48 & -76\end{array}$

$\begin{array}{rr}38 & 21 \\ 114 & 182 \\ -76 & -161\end{array}$

ETP : Évapotranspiration potentielle (formule de Penman-Monteith). 
Tableau II. Valeurs des principaux paramètres culturaux du modèle Epicphase-blé (les numéros entre parenthèses se réfèrent aux équations concernées).

\section{Paramètres communs avec Epic (étalonnage Auzeville)}

Paramètres rendus accessibles à l'utilisateur
Nouveaux paramètres

\begin{tabular}{|c|c|c|c|c|c|}
\hline$k$ & 0,65 & & DO & $\left({ }^{\circ} \mathrm{C} . \mathrm{J}\right)$ & 180 \\
\hline$V\left(m \cdot m^{-1}\right)$ & 1,6 & & D1 & $\left({ }^{\circ} \mathrm{C} . \mathrm{J}\right)$ & 840 \\
\hline$\alpha$ & 5 & & D2 & $\left({ }^{\circ} \mathrm{C} . \mathrm{J}\right)$ & 415 \\
\hline \multirow[t]{14}{*}{$p$} & 0,1 & & D3 & $\left({ }^{\circ} \mathrm{C} . J\right)$ & 435 \\
\hline & & & D4 & $\left({ }^{\circ} \mathrm{C} . J\right)$ & 665 \\
\hline & & (12) & $t$ & & 4 \\
\hline & & (20) & acc & & 5 \\
\hline & & (19) & $\mathrm{Kmx}$ & & 0,2 \\
\hline & & (18) & PSH1 & & $+0,0005$ \\
\hline & & (18) & $\mathrm{PSH} 2$ & & $-0,0008$ \\
\hline & & (18) & PSH3 & & $-0,0100$ \\
\hline & & (18) & PSH4 & & $-0,0025$ \\
\hline & & (18) & PSN1 & & $+0,0005$ \\
\hline & & (18) & PSN2 & & $+0,0015$ \\
\hline & & (18) & PSN3 & & $-0,0010$ \\
\hline & & (18) & PSN4 & & 0 \\
\hline & & (18) & LIMSTR & & 0,8 \\
\hline
\end{tabular}

a Conditions sèches (25), bien alimentées en eau (30) ; b selon les variétés.

\section{En l'absence de facteurs limitants}

\section{Découpage du cycle en phases}

La température de base pour la croissance et le développement $\left(T_{b}\right.$, zéro de végétation) est fixée à $0^{\circ} \mathrm{C}$ pour le blé d'hiver autant pour les parties aériennes que pour les racines (Barraclough, 1984). Sur la base de nos observations et des résultats de l'ITCF dans le sud-ouest de la France (Duboin, 1986), la durée des quatre phases précédentes représente respectivement $27,20,21$ et $32 \%$ du cycle cultural pour des variétés comme Top, Talent, Vizir et Soissons, de précocité voisine. La durée du cycle cultural (STmx, du 15,01 à maturité) est de $2075^{\circ} \times$ jours. La durée semis-levée (D0) est fixée à $180^{\circ} \mathrm{x}$ jours. Une humidité minimale du lit de semence est retenue pour que la levée s'opère $(15 \%)$.

\section{$R \cup E(E q$ 1)}

D'après les auteurs (Monteith, 1977 ; Aase, 1978 ; Gallagher et Biscoe, 1978 ; Green, 1984 ; Gosse et al, 1986 ; Garcia et al, 1988 ; Stapper et Harris, 1989), l'efficience photosynthétique du blé en conditions de croissance potentielle et pour l'ensemble du cycle varie de 2,6 à 3,2 g. $\mathrm{MJ}^{-1}$ (racines comprises). RUE varie cependant au cours du cycle : avant épiaison, Gallagher et Biscoe (1978) obtiennent une valeur de 3,0 g. MJ-1 contre 2,2 pour l'ensemble du cycle. Comme le rappellent ces auteurs, l'activité photosynthétique du couvert diminue dès l'épiaison avec le vieillissement des feuilles et la respiration augmente par unité d'assimilation après l'anthèse avec l'exten- sion des tissus sénescents. La variété et la densité de peuplement ne semblent pas jouer sur RUE (Green, 1989). Aussi on retiendra une valeur de 3,0 g. MJ-1 pour le blé d'hiver (levée-floraison) en bonnes conditions hydriques et azotées. La température optimale de croissance $\left(T_{\text {opt }}\right.$ ) a été fixée à $15^{\circ} \mathrm{C}$ (Williams et al, 1989) : le stress thermique ne joue pas directement sur RUE mais s'applique à la biomasse potentielle.

\section{dRUE (Eq 2)}

En conditions de remplissage favorables, Russell et Wilson (1994) suggèrent une décroissance progressive de RUE entre les stades «épiaison" et «sénescence complète", qui se traduit par une valeur de $\mathrm{d}_{\text {RUE }}$ de 0,25 . Lorsque la demande climatique est plus élevée en fin de cycle (cas du sud-ouest de la France), les capacités de photosynthèse en post-floraison chutent plus rapidement. Aussi, sur la base d'étalonnages antérieurs (Cabelguenne et al, 1990), nous retiendrons une valeur de $d_{R U E}=1$.

\section{$\mathrm{k}(E q 1)$}

La valeur de $k$, coefficient d'extinction de la lumière, dépend de l'angle des feuilles, de leur distribution dans le couvert et de l'inclinaison des rayons du soleil (Fuchs et al, 1984). Des variations de $k$ liées à la densité de peuplement, à la variété et au stade du blé ont été souvent notées (Asrar et Kanemasu, 1985). Les connaissances actuelles ne permettent pas de moduler les valeurs de $k$ par génotype et par phase du cycle (Green, 1989). Aussi, une valeur constante de $k=$ 
0,65, proposée par Monteith (1977), a été retenue pour le blé d'hiver.

\section{Cinétique d'indice foliaire}

La fonction décrivant la cinétique d'établissement de l'indice foliaire pour le blé d'hiver en l'absence de facteurs limitants autres que le rayonnement incident (Eq 3) a été établie après ajustement aux données expérimentales de 1986 (fig 1). La valeur de IF $m x$ dépend de la densité de peuplement (Kiniry et al, 1992) : 300 plantes par $\mathrm{m}^{2}$ permettent d'atteindre un indice foliaire de 7,5 à $50 \%$ du cycle du blé (depuis le 15 janvier), soit peu après le stade gonflement (extension maximale de la dernière feuille).

À partir de suivis de la surface verte menés en 1993 (20 parcelles, cv Soissons), nous avons recherché une valeur moyenne de dIF (Eq 4), paramètre décrivant la vitesse de sénescence physiologique du couvert (fig 2). Selon les parcelles, dIF varie de 0,6 à 1,2 ; aussi, nous retiendrons une valeur unique de dIF $=0,8$ s'appliquant à la surface foliaire à partir de $\mathrm{RST}_{m x}=55$ $\%$ du cycle (soit peu avant l'anthèse).

\section{Progression du front racinaire}

La cinétique de progression des racines en profondeur (Eq 5) est simulée à l'aide des sommes de températures et de la profondeur maximale accessible (en l'absence d'obstacle mécanique). L'ajustement de $V$ s'est appuyé sur des observations endoscopiques (Maertens et Clauzel, 1988 ; Paillard et al, 1992), celui de PMAX sur l'observation de profils de dessèchement du blé à maturité en case profonde (1994). Des valeurs de $P M A X=1,60 \mathrm{~m}$ et $V=1,6 \mathrm{~m} \cdot \mathrm{m}^{-1}$ ont été retenues, assurant un enracinement maximal aux environs de la floraison. La confrontation aux données de Maertens et Clauzel (1988) montre que la simulation de la progression du front racinaire s'écarte encore de la réalité (fig 3 ). Les effets de l'état structural (dégradé après sorgho, favorable après féverole) et du reliquat azoté (faible après sorgho, élevé après féverole) sur la vitesse de progression des racines ne sont pas consi- dérés par le modèle, ce qui accentue les écarts entre valeurs observées et simulées en phase d'installation.

\section{Répartition de la biomasse entre organes (grain, racines)}

EPIC prévoit une décroissance du rapport «biomasse racinaire / biomasse totale" de 0,4 à la levée à 0,2 à maturité. Gregory et al (1978) montrent une augmentation de la part racinaire jusqu'au début du printemps ( $\max =0,34)$ puis une décroissance jusqu'à maturité du blé ( $\min =0,07$ ). En première approche $(\mathrm{Eq} 6)$, en accord avec les options prises par Hansen et al (1991), on retiendra des valeurs de 0,35 (en début de cycle) et de 0,10 (à la récolte du blé), sans tenir compte de l'augmentation initiale du rapport des biomasses observé par Gregory et al (1978).

L'indice de récolte de référence $\left(\mathrm{IR}_{0}\right)$ est celui de peuplements de blé denses, bien alimentés en eau et en azote, et indemnes de maladies ou de verse. Cette valeur est liée au génotype, notamment à la hauteur de paille (Donald et Hamblin, 1976). Pour Soissons, variété à paille courte, une valeur de 0,45 peut être retenue en conditions méridionales (données 1993). Pour Vizir, Top et Talent, une valeur de 0,42 est plus conforme en raison d'une hauteur de paille plus importante (Castillon et al, 1986a)

\section{Fonction de dilution de l'azote}

Pour définir les besoins azotés du blé, Epic simule la dilution de l'azote du peuplement avec la progression du cycle (Jones, 1983) et non avec l'accumulation de biomasse aérienne (Eq 8). L'étalonnage effectué correspond à une teneur de la plante de 2,31 à $50 \%$ du cycle et de $1,34 \%$ à maturité, valeurs observées sur la biomasse totale (racines comprises) (Cabelguenne et al, 1990).

\section{Extraction d'eau en conditions optimales}

À partir des suivis de profils hydriques jusqu'à la date de dessèchement maximal, il a été observé que le blé présente un profil d'extraction de l'eau intermédiaire
Fig 1. Étalonnage de la cinétique d'établissement de l'indice foliaire potentiel ( $\mid F_{p o t}$, $\mathrm{m}^{2} \cdot \mathrm{m}^{-2}$ ) en conditions non limitantes (cv Talent, 1986).

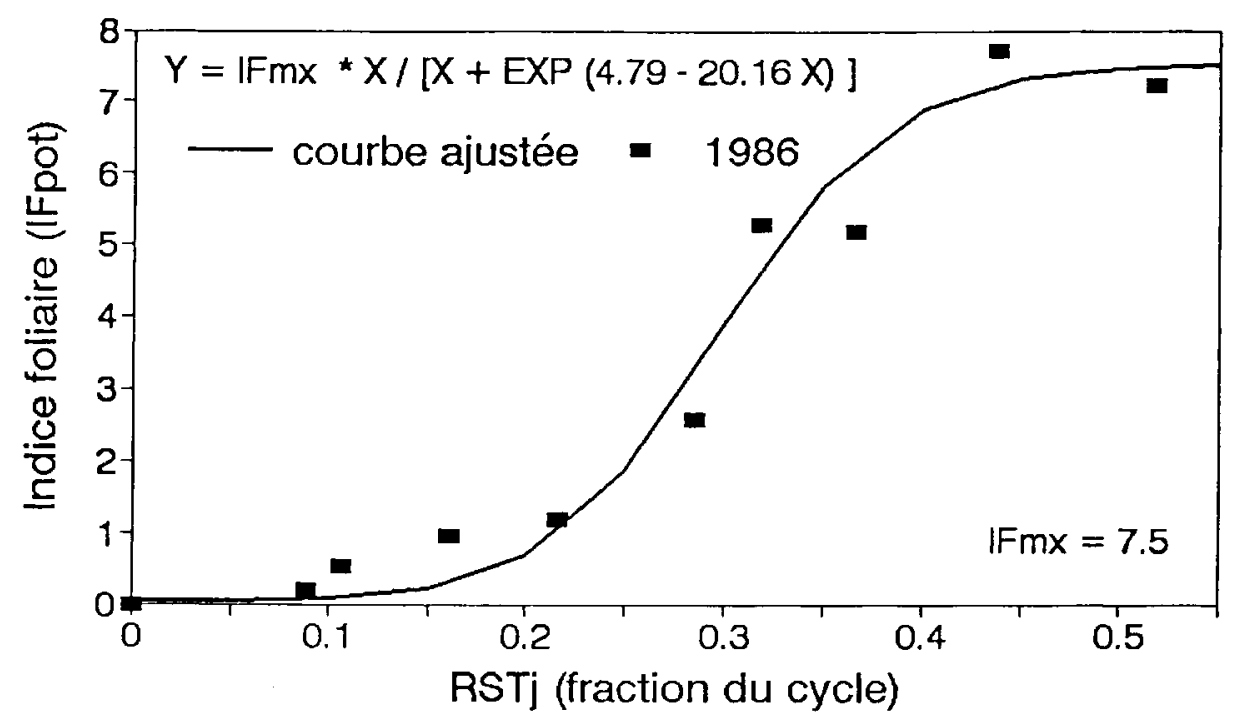




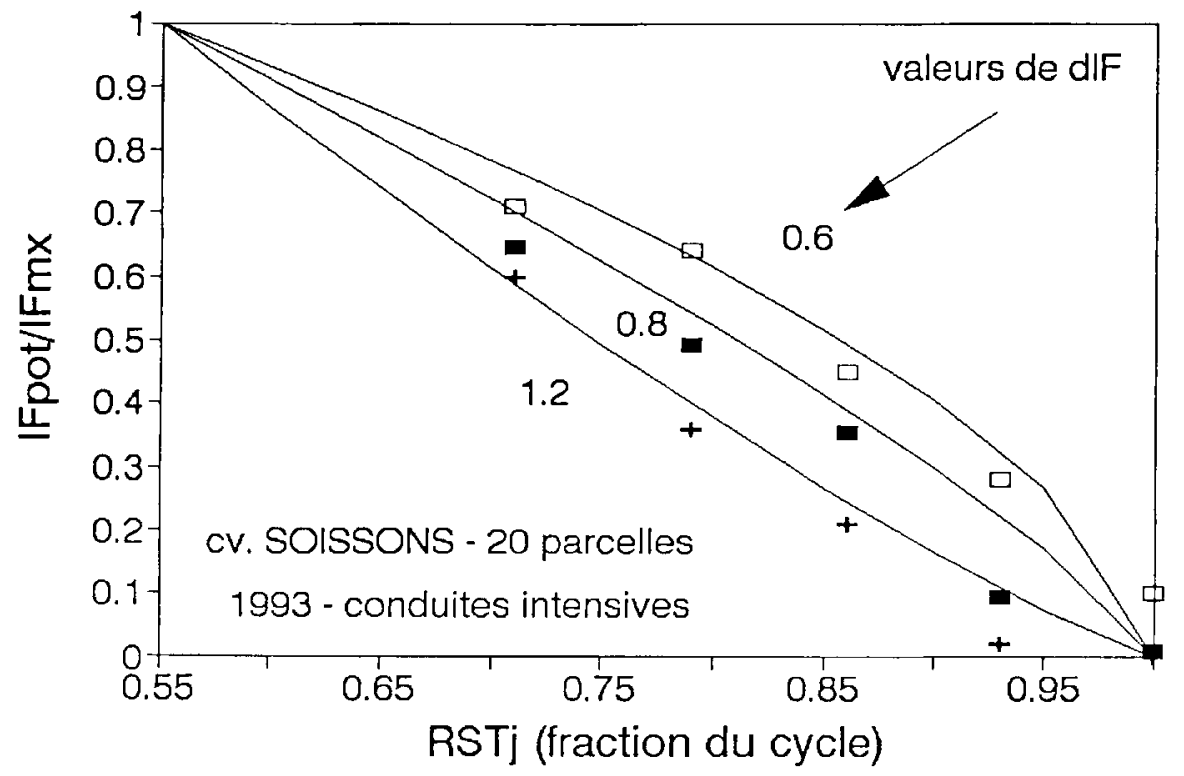

Valeurs observées

moyenne

Fig 2. Étalonnage de la décroissance de l'indice foliaire potentiel $\left(I F_{p o t}\right)$, rapporté à $\mathrm{IF}_{\text {max }}(50 \%$ du cycle), au cours de la sénescence, en l'absence de conditions limitantes ( $\mathrm{cv}$ Soissons, 1993). Les trois courbes représentent les évolutions obtenues avec des valeurs de dIF de $0,6,0,8$ et 1,2 ; les points correspondent aux évolutions extrêmes et moyennes observées en l'absence de facteurs limitants. entre les formes conique du maïs ( $\alpha=10$ ) et cylindrique du tournesol $(\alpha=0,1)$. Une valeur d' $\alpha=5$ en sol profond est retenue pour le blé alors qu'en sol superficiel (lysimètres 1994) on observe une extraction plus cylindrique $(\alpha=1)$. De même, on considère qu'en sol profond la compensation par l'enracinement profond est partielle $(p=0,1)$ alors qu'en sol superficiel $(<1 \mathrm{~m})$ on peut faire l'hypothèse d'une forte colonisa- tion racinaire en profondeur et d'un fonctionnement homogène du système racinaire $(p=1)$.

\section{Augmentation du besoin hydrique}

Epicphase autorise I'ETM du blé à dépasser de $20 \%$ I'ETP $\left(\mathrm{K}_{m x}=0,2\right)$ entre les stades «2 nœuds» et "épiaison +1 mois» (Bouthier et Bonnefoy, 1993), soit entre 35 et $65 \%$ du cycle.

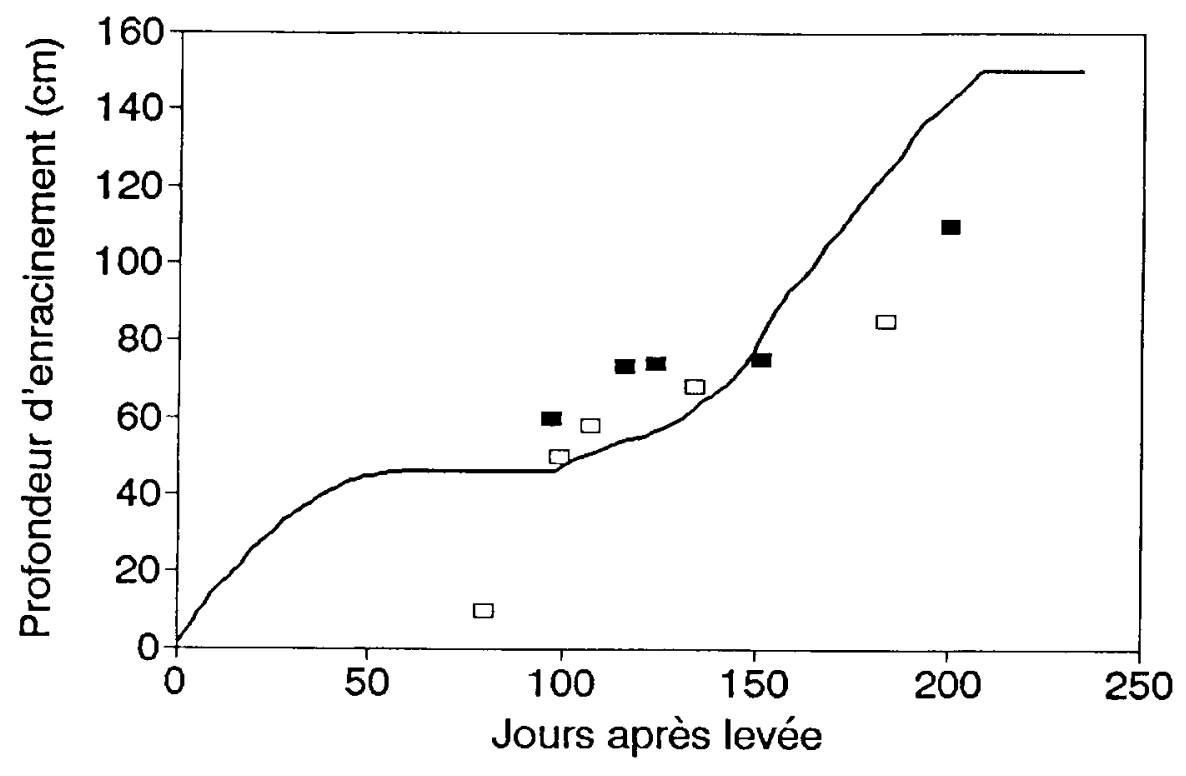

$-\operatorname{Sim}=$ Obs1 ㅁ OBS2

OBS 1 = Blé sur Tournesol ou Féverole

OBS 2 = Blé sur Mais ou Sorgho

Fig 3. Étalonnage au champ de la progression de l'enracinement en profondeur après différents précédents (cv Talent, 1985) ; données observées par endoscopie. 


\section{En présence de stress hydriques ou azotés}

\section{Effet du stress hydrique sur la vitesse de sénescence foliaire}

Sur blé d'hiver, nous avons testé l'intèrêt de cette procédure à partir de deux situations diversement alimentées en eau, ayant fait l'objet d'un suivi d'évolution de la surface verte après floraison (fig 4). En 1990, une valeur d'acc $=5$ permet de rendre compte des diverses cinétiques de sénescence sous l'effet du stress hydrique. En l'absence d'irrigation, la surface verte est quasi nulle à $80 \%$ du cycle, alors qu'en conditions de bonne alimentation en eau la sénescence n'est complète qu'à $95 \%$ du cycle.

\section{Effet du stress hydrique sur l'indice de récolte}

On se reportera à l'article de Debaeke et al (1996) pour l'étalonnage de ce module en lysimètres.

\section{Effet du stress azoté sur l'indice de récolte}

Les données de la littérature (Donald et Hamblin, 1976), d'une part, nos données expérimentales (témoins non fertilisés), d'autre part, montrent un accroissement de l'indice de récolte du blé (en moyenne $+0,03$ en 1993) avec le défaut d'alimentation azotée, effet qui serait renforcé par le stress hydrique (Donald et Hamblin, 1976). Cet effet global a été réparti selon les phases du cycle du blé : en phases 1 et 2 , une carence azotée augmente l'indice de récolte (rationnement végétatif) ; en phase 3 , une carence pénalise davantage le nombre de grains que la biomasse totale et donc diminue IR ; enfin, en phase 4, IR n'est pas affecté par le manque d'azote. Les valeurs des pentes de variation de IR $\left(\mathrm{PSN}_{i}\right)$ en fonction du nombre de jours de stress azoté figurent au tableau II.

\section{Analyse de sensibilité du modèle Epicphase}

Les principaux paramètres culturaux du tableau II ont fait l'objet d'une analyse de sensibilité dans le cadre d'une culture de blé non irriguée soumise à une série climatique de 11 ans (1984-1994) et à un régime pluviométrique très restreint. La variation relative moyenne du rendement consécutivement à une modification de $\pm 10 \%$ de la valeur de chaque paramètre a été classée à la figure 5 . $\mathrm{IR}_{0}$, RUE et, dans une moindre mesure, $\mathrm{ST}_{m x}$, $\mathrm{RST}_{m x}$ et $t$ sont les paramètres les plus sensibles d'Epicphase. En condition sèche, le rendement est particulièrement sensible aux nouveaux paramètres de consommation hydrique $(t, p, \alpha)$ et de réponse de l'indice récolte au stress (PSH3, PSH4).

\section{RÉSULTATS DE L'ÉVALUATION D'ÉPICPHASE}

\section{Validation du découpage en phases}

Les durées de phases simulées par le modèle et relevées au champ ont été comparées pour les variétés Talent (1984-1986), Vizir (1987-1991) et Soissons (1992-1993). La durée de la phase "semis-levée» est assez bien simulée par Epicphase sauf pour les conditions de sécheresse automnale de 1988, 1989 et 1992, où le modèle se révèle plus optimiste (fig 6a). L'écart moyen (RMSE) est de 5,5 jours. La durée de la phase «semi-épi $1 \mathrm{~cm}$ » est simulée avec un écart moyen de 84 degrés.jours pour des dates de semis assez étalées (fig 6 b) et la durée
Fig 4. Étalonnage de la vitesse de sénescence du couvert selon la contrainte hydrique subie par le blé d'hiver (cv Vizir, 1990) : conduite en sec (précédent sec) et irrigation $75 \mathrm{~mm}$ (précédent irrigué $250 \mathrm{~mm}$ ).

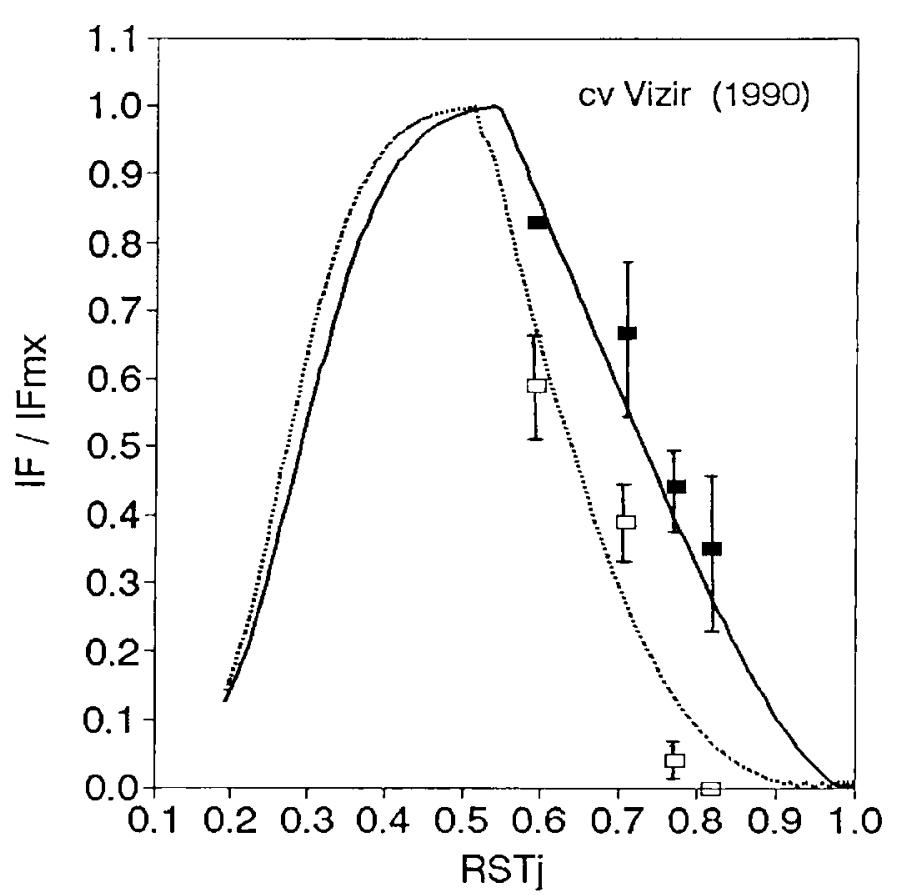

Non Irrigué (sim)

Non irrigué (obs)

Irrigué $75 \mathrm{~mm}$ (sim)

Irrigué $75 \mathrm{~mm}$ (obs) 


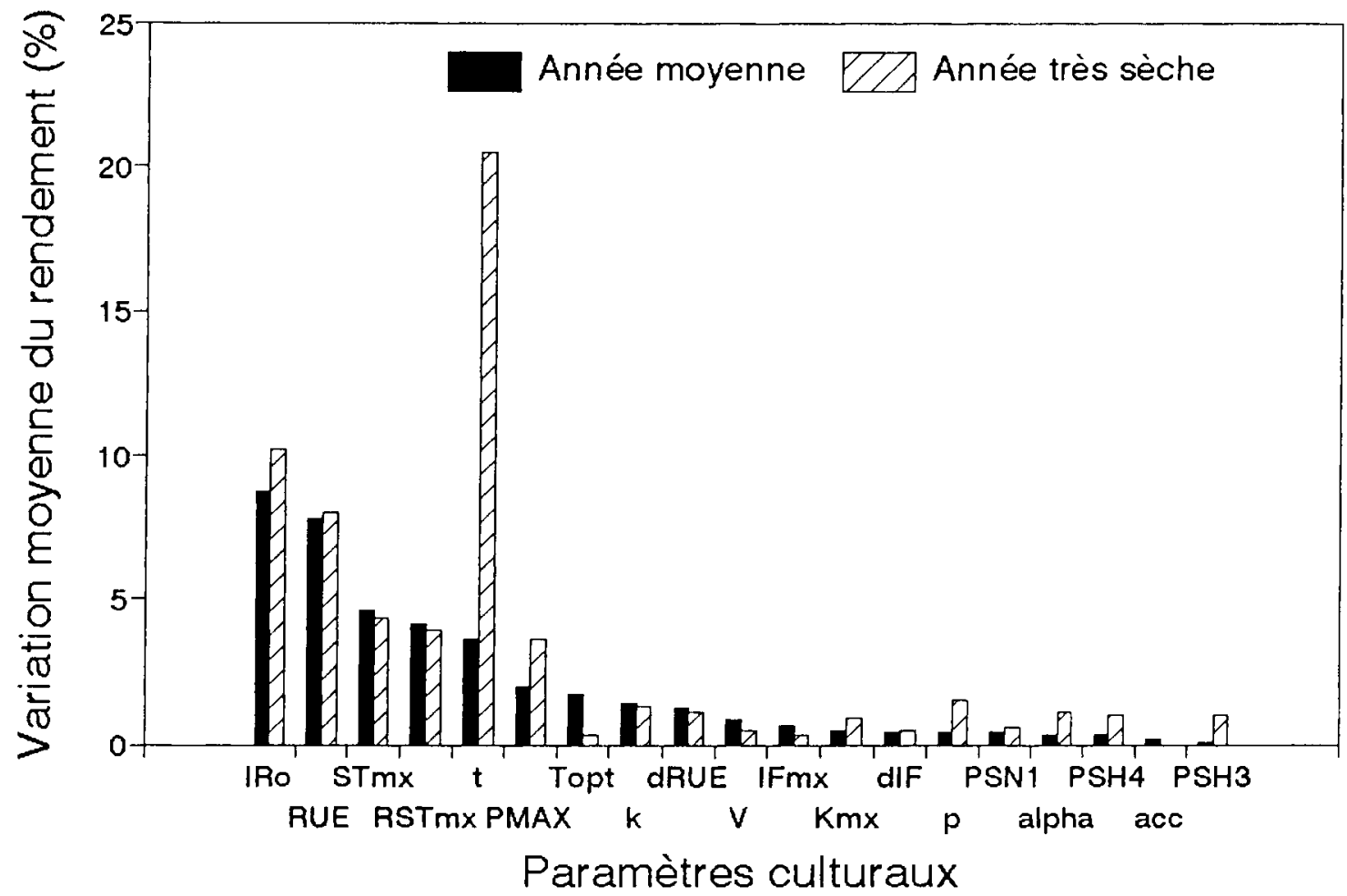

Fig 5. Analyse de sensibilité du modèle Epicphase : variation moyenne du rendement du blé pour une variation de $+10 \%$ et $-10 \%$ de la valeur de chaque paramètre en année moyenne (1984-1994) et en condition très sèche.

«semis-épiaison» est simulée avec un écart de 59 degrés.jours (fig 6c).

\section{Simulation du fonctionnement du blé en l'absence de stress hydrique}

\section{Biomasse aérienne}

Une simulation satisfaisante de l'accumulation de biomasse aérienne (MST, t.ha ${ }^{-1}$ ) est obtenue en 1993 pour la variété Soissons en conduite intensive après tournesol (fig 7). En l'absence d'azote, le modèle surestime la croissance printanière, en raison d'une minéralisation trop rapide des résidus de récolte du tournesol.

\section{Indice de récolte}

L'indice de récolte $(0,38$ à 0,50$)$ est bien simulé en 1984 (cv Talent) pour une gamme d'alimentation azotée (fig 8 ); ceci valide globalement l'étalonnage des pentes de sensibilité d'IR au stress azoté par phase, évoqué plus haut.

\section{Consommation hydrique}

Enfin, à partir d'évapotranspiromètres bien alimentés en eau (cv Top 1982), on montre que le modèle rend bien compte des variations de consommation à l'ETM observées sur une base décadaire (fig 9).

\section{Validation du bilan hydrique en lysimètres}

Les grandes cases lysimétriques (à drainage contrôlé) et les petits fûts variant par leur texture, leur profondeur et les régimes d'apport d'eau ont été utilisés pour valider le bilan hydrique de la culture de blé (cv Top 1982). Toutes situations confondues, le modèle permet de simuler la consommation hydrique du blé avec une erreur de $64 \mathrm{~mm}$ (soit $14 \%$ de la valeur moyenne observée) (fig 10). À partir d'une case sèche peu profonde (cv Vizir 1991), on montre que la dynamique d'évolution des réserves en eau du sol est correctement simulée, notamment en phase de forte dessiccation (fig 11).

\section{Validation globale au champ (1989 et 1990)}

L'état du profil de sol à la récolte du précédent cultural doit être estimé pour initialiser le modèle sur chacune des 50 parcelles du test ( $N$ et $\mathrm{ON}$ ). Étant donné la gamme de situations expérimentales, nous avons procédé en trois étapes : i) 

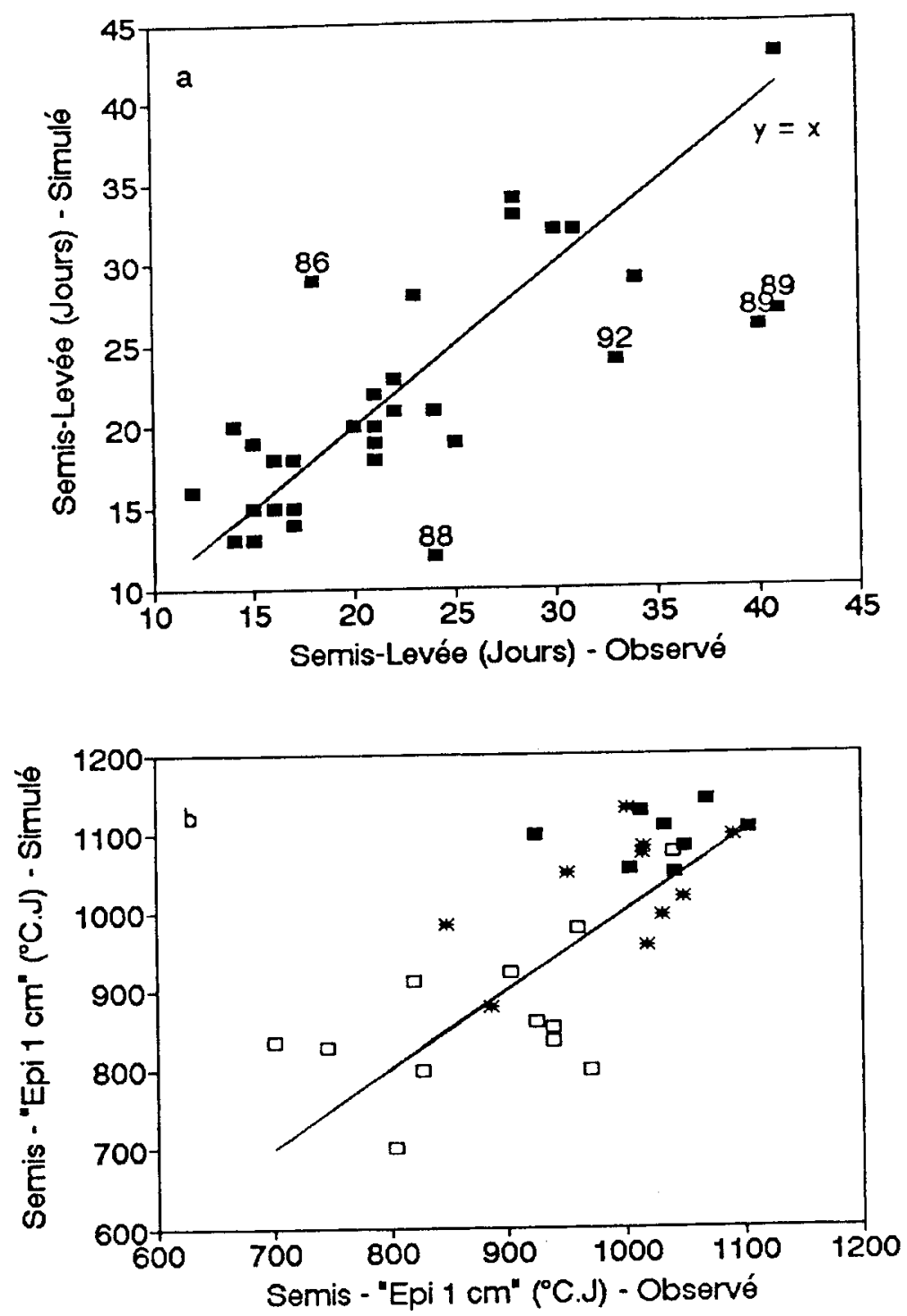

- semis < 5 Nov. * 5 Nov au 25 Nov $\square>25$ Nov

Fig 6. Validation de la durée des phases phénologiques : a. semislevée (jours) ; b. semis-stade épi $1 \mathrm{~cm}$ (degrés.jours) ; c. semisépiaison (degrés.jours).

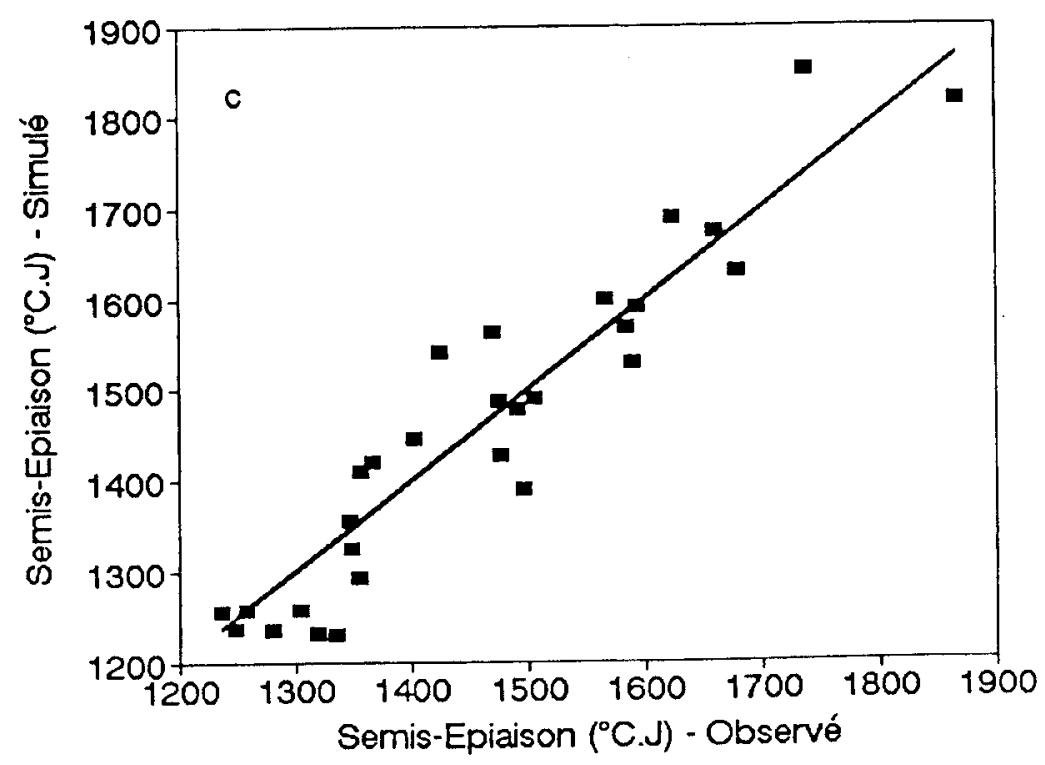



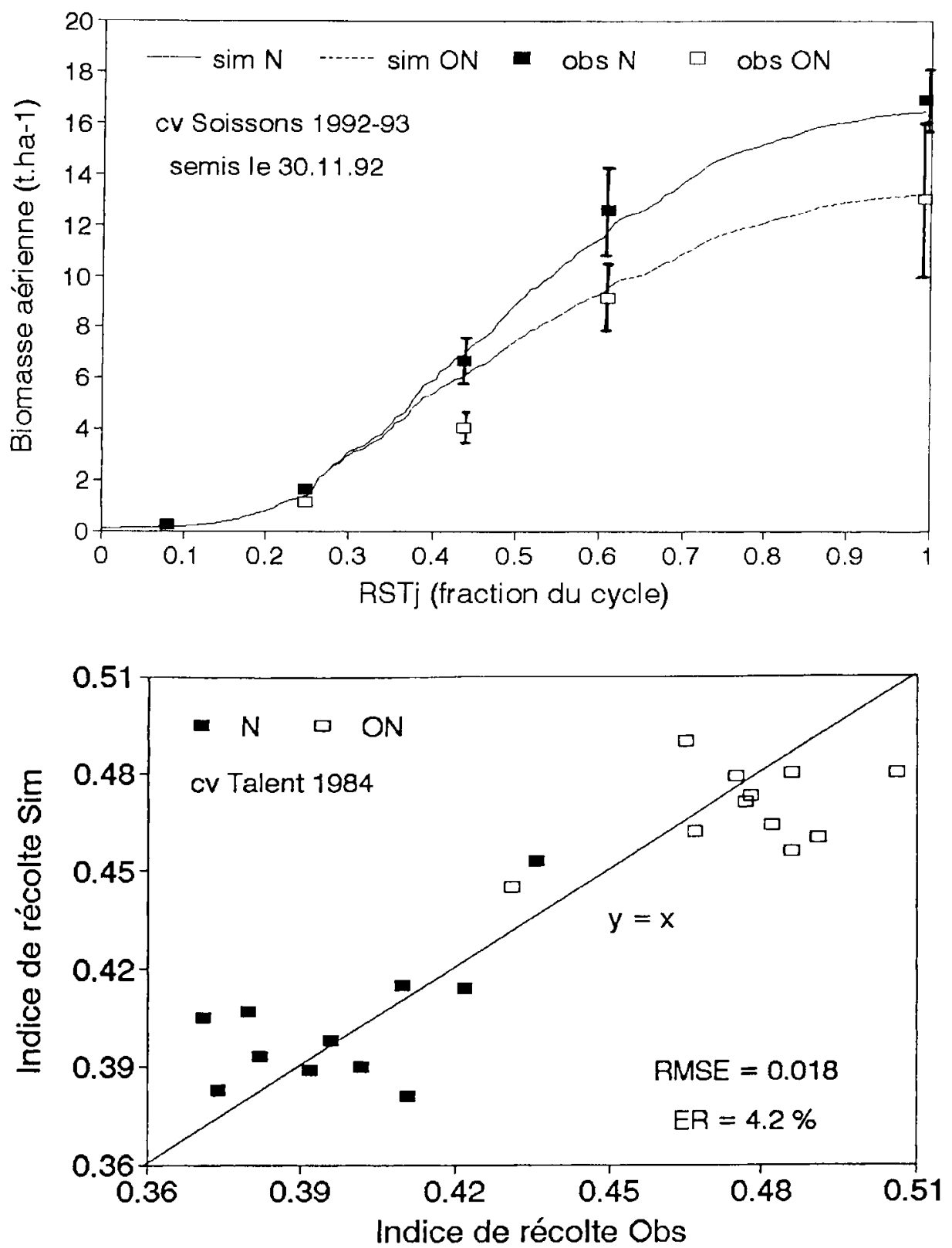

Fig 7. Validation de la dynamique de croissance du blé en l'absence de contrainte hydrique (cv Soissons 1993) : effet de l'alimentation azotée.
Fig 8. Validation de l'indice de récolte du blé (cv Talent 1984) avec (ON) ou sans défaut d'alimentation azotée $(\mathrm{N})$. Les parcelles ON ne reçoivent pas d'engrais azoté en 1984 ; les parcelles $\mathrm{N}$ reçoivent entre 120 et $210 \mathrm{~kg}$ d'azote par ha. estimation de l'état de remplissage initial de la réserve du sol par groupes de précédents (nature de la culture, modalités d'irrigation) à partir des mesures neutroniques; ii) estimation de la quantité de résidus laissée par le précédent sur la base de la matière sèche aérienne non exportée à la récolte (mesurée) augmentée de $20 \%$ pour la masse racinaire ; iii) estimation de la quantité d'azote minéral présente à l'entrée de l'hiver par la méthode du bilan prévisionnel régionalisée (Castillon et al, 1986b).

\section{Cas d'une année de forte sécheresse en post-épiaison (1989)}

La valeur de la biomasse aérienne totale (MST) est prévue par le modèle avec un écart moyen de 1,5 t.ha-1, soit $13,3 \%$ de la moyenne observée (fig 12a). On note une plus forte dispersion des points pour les valeurs intermédiaires (témoins $O N$ ). L'indice de récolte (IR) est assez bien simulé par le modèle avec un écart relatif de $9,4 \%$ et une certaine tendance à la surestimation par le modèle (fig 12b). L'effet positif d'une carence azotée précoce sur l'établissement d'IR est bien figuré ici. En conséquence, le rendement du blé (RDT) soumis à une forte sécheresse de post-épiaison est simulé avec une erreur moyenne de 7,6 q.ha-1 (fig 12c). La teneur en azote de la biomasse aérienne est prévue avec un écart relatif de $13 \%$ (fig 12d); le modèle sous-estime cette variable en conditions de faible alimentation azotée. 
Fig 9. Validation de l'évapotranspiration maximale (ETM, $\mathrm{mm}$ ) du blé (évapotranspiromètres, cv Top, 1982) sur une base décadaire.
Fig 10. Validation de l'eau consommée par le blé (ETR, $\mathrm{mm}$ ) en cases lysimétriques et en petits fûts (cv Top 1982) pour des textures et des régimes d'irrigation variés.
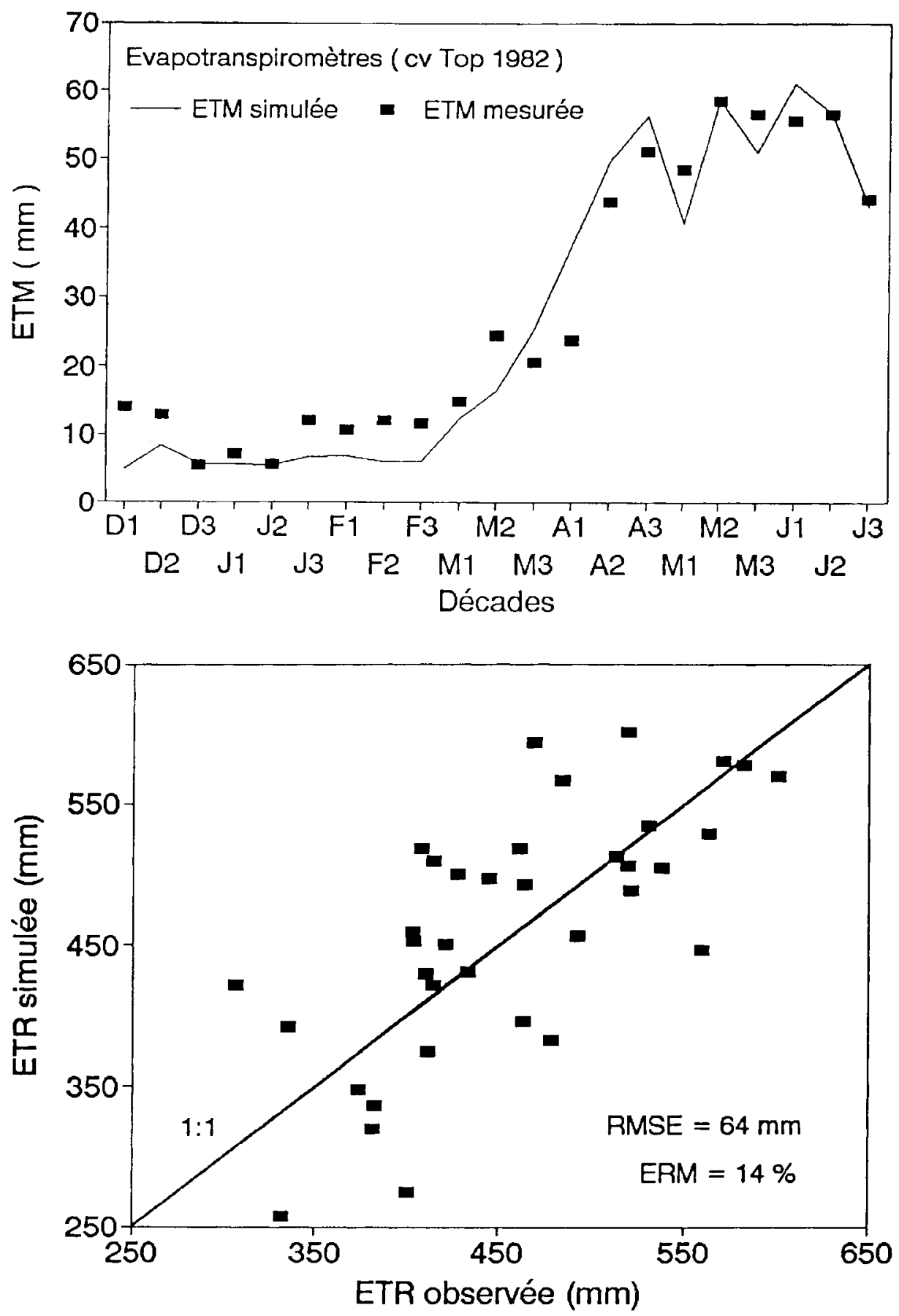

\section{Cas d'une année de sécheresse précoce (1990)}

La performance du modèle est du même ordre en 1990. Ainsi, MST est prévue avec un écart moyen de 1,4 t.ha $^{-1}$, soit également $13,3 \%$ de la moyenne des observations (fig 13a). L'effet déterminant de l'irrigation en cours de montaison est bien pris en compte par le modèle. IR est assez bien simulé avec un écart relatif de $8,1 \%$ (fig 13b). Les valeurs d'IR diffèrent peu entre niveaux de fumure azotée, en accord avec l'offre du sol élevée quel que soit le précédent (reli- quats élevés, hiver doux et sec). En conséquence, le rendement du blé soumis à une sécheresse précoce (dès le stade "épi $1 \mathrm{~cm}$ ") est simulé avec un écart moyen de 6,1 q.ha-1 (fig 13c). Enfin, la teneur en azote est assez bien simulée (écart relatif de 15,5\%) (fig 13d).

\section{Comparaison de la performance des modèles Epic et Epicphase}

Le modèle Epicphase permet d'améliorer la simulation de RDT, MST, IR, NABS/MST, ETR et de l'efficience de l'eau (RDT/ETR et MST/ETR) 


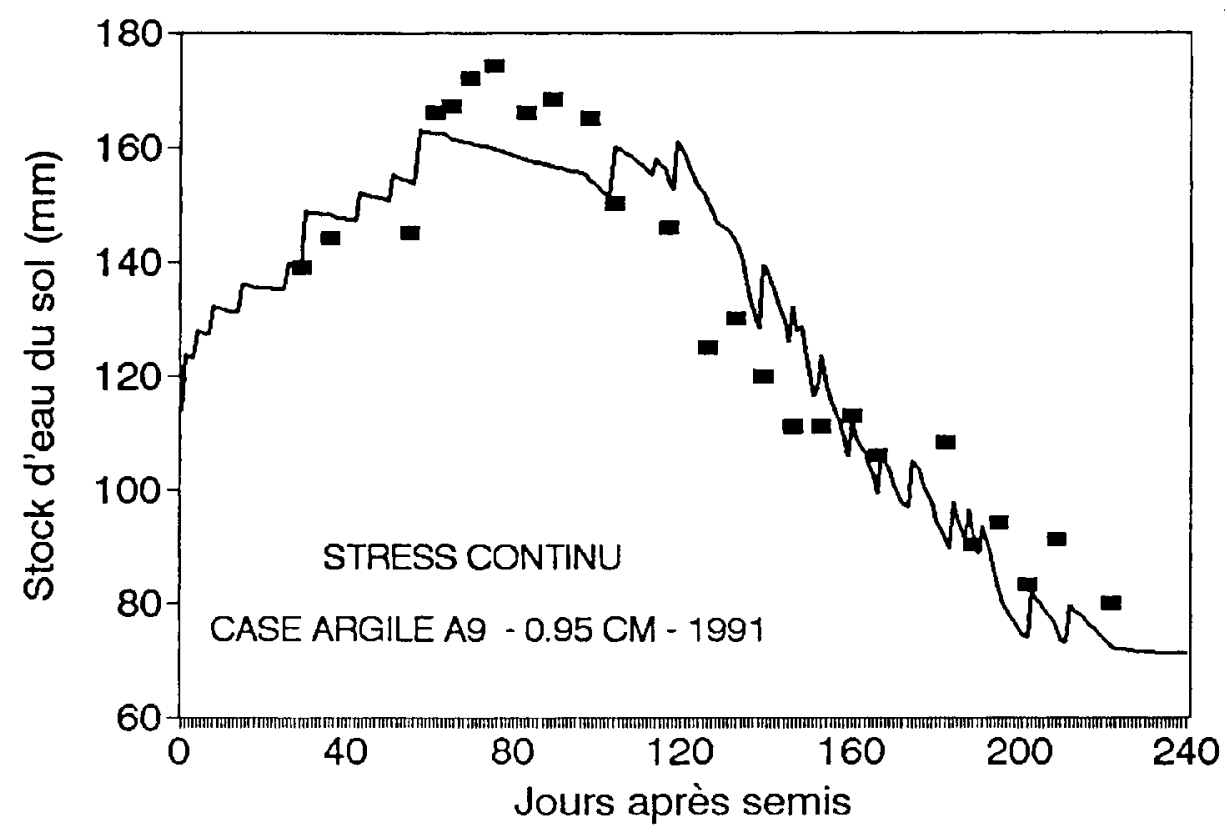

- observé — simulé
Fig 11. Validation de l'évolution du stock d'eau du sol (mm) au cours du cycle du blé, en case peu profonde et peu irriguée (cv Vizir 1991). a.

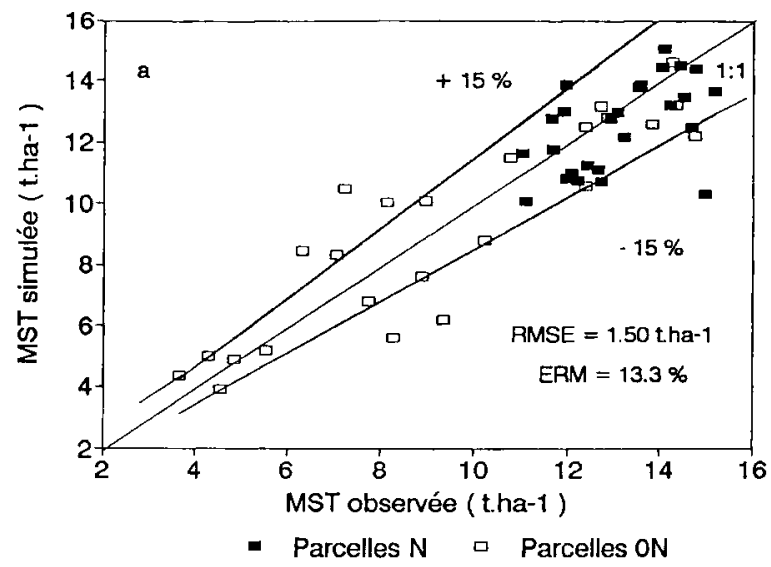

c.

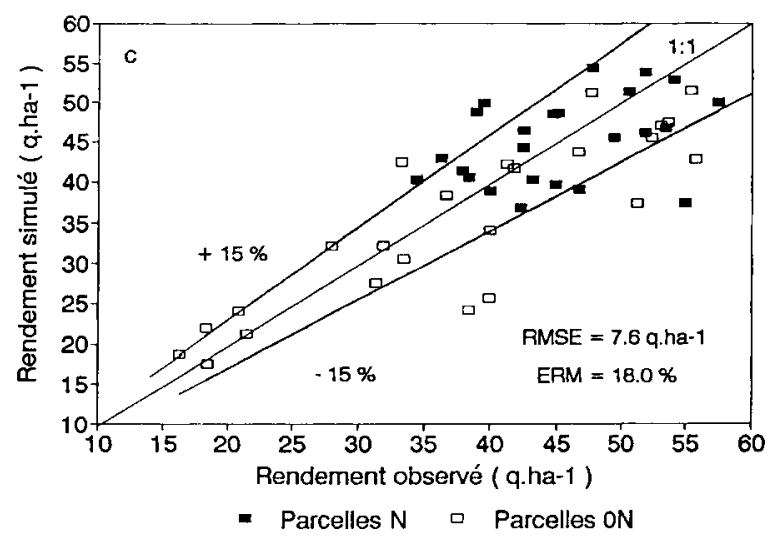

b.

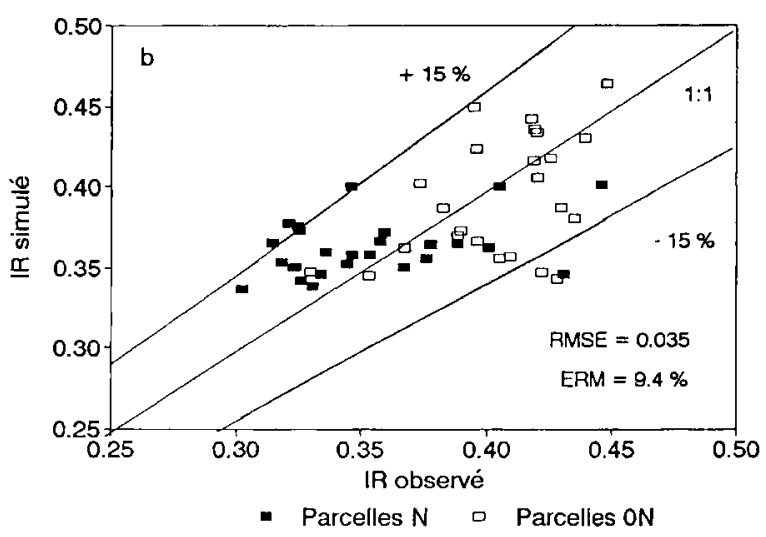

d.

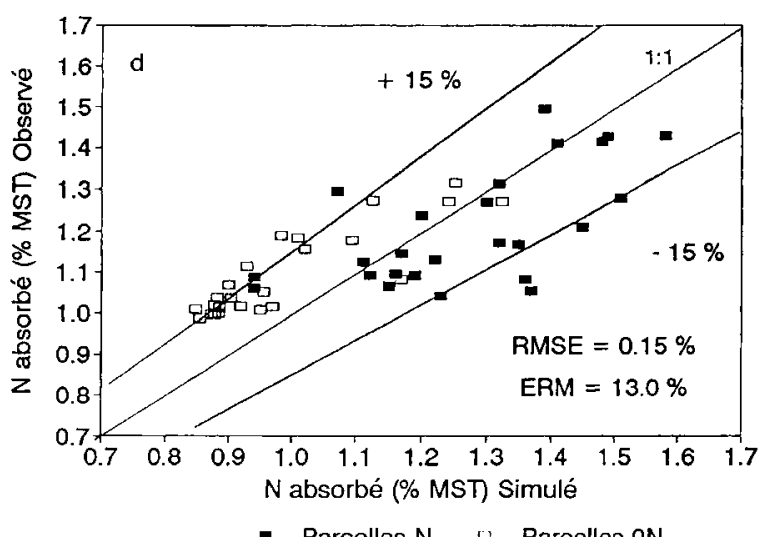

- Parcelles $N$ u Parcelles ON

Fig 12. Test au champ du modèle Epicphase-Blé (cv Vizir 1989) : a. biomasse aérienne totale (MST, tha- ${ }^{-1}$ ) ; b. indice de récolte (IR); c. rendement (RDT, q.ha-1) ; $d$. teneur en azote de la biomasse aérienne totale $(\mathrm{N} \%)$. 
en conditions d'alimentation hydrique limitante (tableau III). L'indicateur RMSE est en effet toujours plus faible pour Epicphase que pour Epic sauf en 1989 pour MST. L'amélioration est particulièrement nette pour IR tant en 1989 qu'en 1990 et pour MST en 1990 dans le cas d'une sécheresse courant montaison.

\section{Effet d'une sécheresse continue}

Le modèle a été testé sur des cases argileuses et limoneuses peu profondes $(0,95 \mathrm{~m})$ conduites en stress continu depuis la levée (cv Vizir 1991) ou à partir du redressement (cv Soissons 1994). Le stress brutal en 1994 (forte biomasse au redressement) s'est traduit par une chute d'IR plus prononcée qu'en 1991 (Debaeke et al, 1996), dont le modèle a bien tenu compte (tableau IV). Les valeurs de MST et par consé-

a.

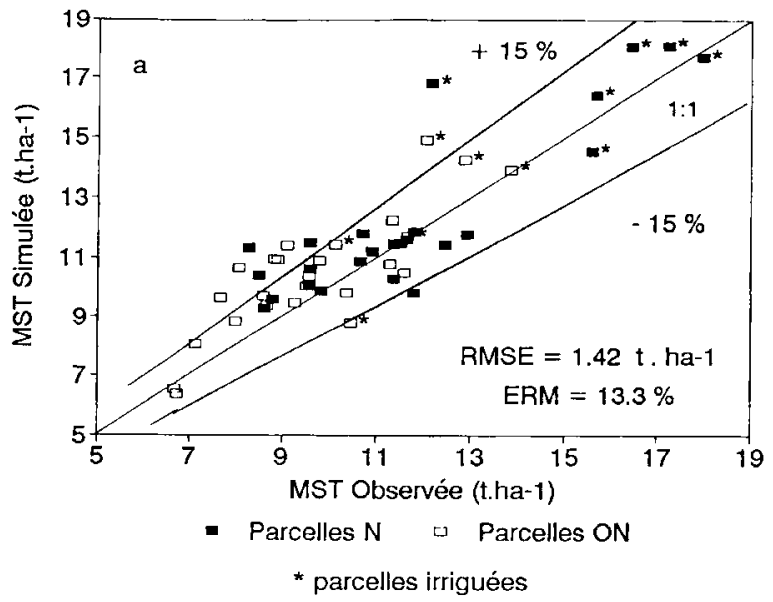

c.

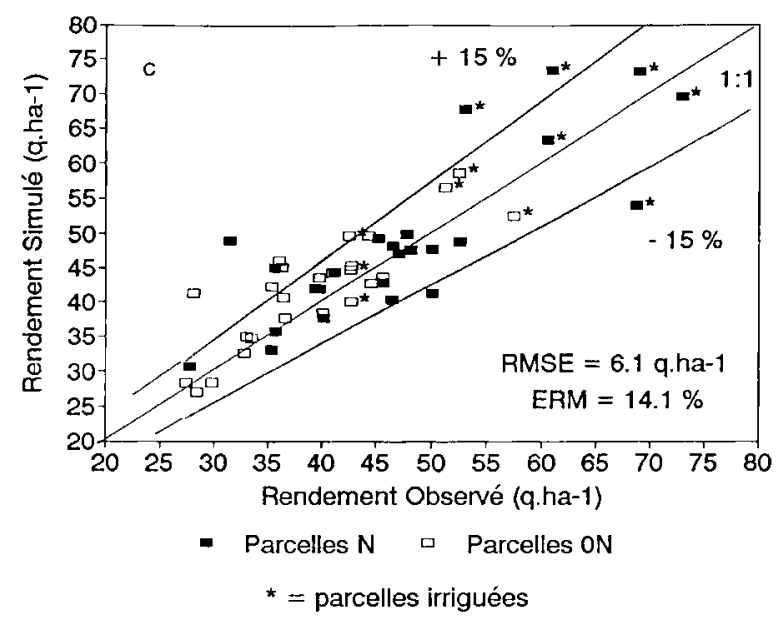

quent de RDT sont assez mal prévues en 1994 traduisant probablement des processus adaptatifs. La variété Soissons serait assez tolérante à la sécheresse, au vu de son bon comportement en 1989 et 1990 dans le sud-ouest de la France. L'efficience pour la production de grain est par ailleurs sous-estimée en conditions de forte sécheresse, comme cela s'observait déjà au champ. En 1991, le rendement est bien simulé in fine mais par le biais de phénomènes de compensation non conformes à la réalité.

\section{DISCUSSION ET CONCLUSIONS}

Le modèle Epicphase a été testé en sol profond pour une variété de blé d'hiver (cv Vizir), dans une gamme limitée de conditions climatiques (deux années). La gamme étendue de conditions d'alimentation hydrique (irrigation, scénarios de

b.

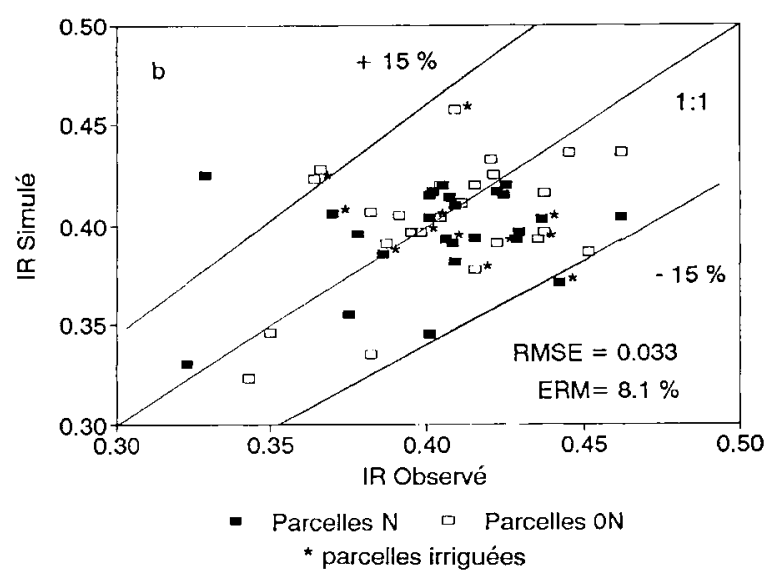

d.

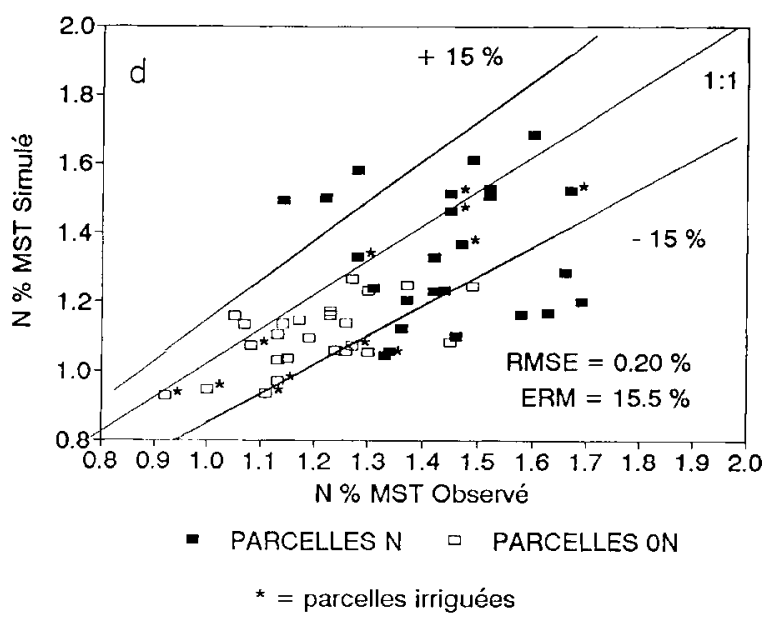

Fig 13. Test au champ du modèle Epicphase-Blé (cv Vizir 1990) : a. biomasse aérienne totale (MST, t.ha-1); b. indice de récolte $(\mathrm{IR})$; c. rendement (RDT, q.ha-1) ; d. teneur en azote de la biomasse aérienne totale $(\mathrm{N} \%)$. Les valeurs de $\pm 15 \%$ s'appliquent à la droite $y=x$. 
Tableau III. Comparison des performances des modèles Epic et Epicphase en conditions hydriques limitantes.

\begin{tabular}{ccccc} 
Observation & Epic & \multicolumn{2}{c}{ Epicphase } \\
Moyenne & Moyenne & RMSE ERM (\%) Moyenne RMSE ERM
\end{tabular}

1989

\begin{tabular}{|c|c|c|c|c|c|c|c|}
\hline RDT (t.ha $\left.a^{-1}\right)$ & 4,24 & 4,82 & 1,04 & 24,6 & 4,03 & 0,76 & 18,0 \\
\hline MST (t.ha-1) & 11,28 & 11,25 & 1,34 & 11,9 & 10,89 & 1,50 & 13,3 \\
\hline IR & 0,378 & 0,428 & 0,078 & 20,6 & 0,370 & 0,034 & 9,0 \\
\hline NABS/MST (\%) & 1,17 & 1,10 & 0,020 & 12,4 & 1,14 & 0,022 & 13,0 \\
\hline \multicolumn{8}{|l|}{990} \\
\hline RDT (t.ha-1) & 4,34 & 5,65 & 1,56 & 35,9 & 4,54 & 0,61 & 14,1 \\
\hline MST (t.ha-1) & 10,74 & 13,20 & 2,49 & 23,2 & 12,00 & 1,42 & 13,3 \\
\hline IR & 0,405 & 0,428 & 0,051 & 12,6 & 0,399 & 0,033 & 8,1 \\
\hline NABS/MST (\%) & 1,32 & 1,10 & 0,275 & 20,8 & 1,22 & 0,204 & 15,5 \\
\hline \multicolumn{8}{|l|}{ 989/1990 } \\
\hline ETR $(\mathrm{mm})$ & 410 & 434 & 38,6 & 9,4 & 410 & 30,2 & 7,4 \\
\hline RDT/ETR $(\mathrm{kg} / \mathrm{mm})$ & 11,1 & 15,3 & 4,7 & 42,4 & 12,3 & 1,3 & 10,9 \\
\hline MST/ETR $(\mathrm{kg} / \mathrm{mm})$ & 31,6 & 34,3 & 7,7 & 24,5 & 32,2 & 6,2 & 19,5 \\
\hline
\end{tabular}

${ }^{a}\left(\right.$ t.ha $\left.{ }^{-1}\right),{ }^{b}(\%),{ }^{c}(\mathrm{~mm}),{ }^{d}(\mathrm{~kg} / \mathrm{mm})$.

Tableau IV. Simulation de l'effet d'une sécheresse continue sur le blé d'hiver (cv Vizir 1991 et Soissons 1994) - cases argileuses et limoneuses.

\begin{tabular}{|c|c|c|c|c|c|c|c|}
\hline Cases & & $\begin{array}{c}E T R \\
(\mathrm{~mm})\end{array}$ & $\begin{array}{c}M S T \\
\left(t . h a^{-1}\right)\end{array}$ & $I R$ & $\begin{array}{c}R D T \\
\left(t h a^{-1}\right)\end{array}$ & $\begin{array}{c}\text { RDT/ETR } \\
\left(\mathrm{kg} \cdot \mathrm{mm}^{-1}\right)\end{array}$ & $\begin{array}{l}\text { MST/ETR } \\
\left(\mathrm{kg} \cdot \mathrm{mm}^{-1}\right)\end{array}$ \\
\hline Limon 1991 & OBS & 193 & 7,71 & 0,285 & 2,20 & 11,4 & 39,9 \\
\hline (34\% ETM) & SIM & 253 & 8,39 & 0,269 & 2,27 & 9,0 & 33,2 \\
\hline Argile 1991 & OBS & 194 & 8,37 & 0,307 & 2,57 & 13,2 & 43,1 \\
\hline (29 \% ETM) & SIM & 252 & 8,57 & 0,285 & 2,43 & 9,6 & 34,0 \\
\hline Limon 1994 & OBS & 257 & 13,62 & 0,193 & 2,63 & 10,2 & 53,0 \\
\hline (51\% ETM) & SIM & 200 & 9,58 & 0,203 & 1,95 & 9,8 & 47,9 \\
\hline Argile 1994 & OBS & 270 & 11,32 & 0,243 & 2,75 & 10,2 & 41,9 \\
\hline (54\% ETM) & SIM & 191 & 8,78 & 0,201 & 1,76 & 9,2 & 46,0 \\
\hline
\end{tabular}

sécheresse, précédent irrigué ou non) et azotée (précédent cultural, apports de 0 à $210 \mathrm{~kg} \cdot \mathrm{ha}^{-1}$ ) a permis de tester l'aptitude du modèle à intégrer des interactions eau-azote complexes. Chaque année, 50 situations ont été soumises au test et l'on s'est refusé à écarter une situation sans raison objective (verse par exemple). Dans ces conditions d'extrême variabilité liée au précédent cultural (quantité de résidus de récolte, reliquats d'azote en entrée d'hiver, état de dessiccation ini- tial du profil) et à la conduite annuelle (date de semis, fractionnement et dose d'engrais azoté ou d'irrigation), l'écart relatif de rendement entre observation et simulation reste inférieur à $15 \%$ dans $76 \%$ des situations en 1989 et $78 \%$ en 1990. La performance du modèle Epicphase rejoint celle des modèles spécifiques au blé, qui permettent une estimation du rendement avec un écart de $12-15 \%$ en conditions non limitantes (Porter et al, 1993). 
L'écart résiduel entre simulation et observation (8 à $18 \%$ selon les variables et les années) doit être recherché dans quatre directions :

\section{Les données expérimentales qui servent au test du modèle sont insuffisamment précises pour permettre une convergence plus étroite entre les deux valeurs}

Cette hypothèse ne peut être écartée. En effet, le coefficient de variation intra-parcellaire moyen sur le rendement est de $10 \%$ en 1990 , avec une expression plus forte en $\sec (4$ à $21 \%$ ) qu'en irrigué ( 3 à $12 \%)$. L'hétérogénéité du milieu, forte en sol alluvial et renforcée par le déficit hydrique de l'année, est un frein à l'application de modèles ponctuels à l'échelle d'une parcelle donnée. II paraît alors logique de considérer le modèle comme validé lorsque la valeur simulée ne diffère pas de plus de $15 \%$ de la valeur observée.

Une incertitude demeure sur les stocks initiaux d'eau et d'azote, en raison pour partie de la variabilité du milieu mais, également, de la méthode d'estimation utilisée. Le bilan prévisionnel de l'azote permet de prévoir la dose d'engrais optimale à $40 \mathrm{~kg} \cdot \mathrm{ha}^{-1}$ près dans $85 \%$ des situations testées dans le sud-ouest (Castillon et al, 1986b).

\section{Le modèle ne simule pas l'ensemble des facteurs limitants de la culture de blé}

Le diagnostic cultural pratiqué sur la culture de blé en 1989 et 1990 a révélé plusieurs facteurs limitants que le modèle ne pouvait reconnaître:

- les hautes températures en phase de remplissage (en 1989,18 jours où la température maximale a été supérieure à $25^{\circ} \mathrm{C}$ ), dont on connaît les conséquences négatives sur le remplissage du grain (Gate et Massé, 1990), indépendamment des niveaux d'alimentation hydrique. Si le modèle intègre l'effet des hautes températures sur le fonctionnement photosynthétique de la culture, il ne simule pas les effets d'échaudage par le biais de l'indice de récolte. Si le déficit hydrique et les hautes températures sont souvent associés au champ en phase de remplissage (1989), ces effets ont pu être dissociés lors de l'étalonnage du modèle en lysimètres, dans la mesure où seuls trois jours très échaudants (Tmaxi $>28^{\circ} \mathrm{C}$ ) ont été rencontrés en 1991 lors du remplissage ;
- en 1990, les semis précoces ont subi en cours de montaison un gel brutal qui a affecté la fertilité de l'épi mais peu la biomasse végétative. Si le modèle simule l'effet du gel sur la croissance du blé (Williams et al, 1989), il ne prévoit pas d'effet sur l'indice de récolte. Une modélisation simple de l'effet des températures extrêmes sur la formation d'IR devrait être recherchée (en particulier, l'effet de basses températures aux environs de la méiose qui affecte le taux de nouaison) ;

- si les conditions de sécheresse ont limité l'extension des maladies, des attaques précoces d'oïdium et des symptômes de virose ont pu être observés en 1990 sur certaines parcelles après I'hiver très doux. Ceci souligne l'intérêt d'intégrer par culture (et plus spécialement sur blé) une estimation complémentaire des pertes de rendement liées aux maladies en fonction de symptômes observés en cours de culture. Ainsi, une accélération de la sénescence foliaire et (ou) une baisse d'indice de récolte pourraient rendre compte des effets de la rouille brune.

\section{Le modèle simule imparfaitement certains processus}

En dépit d'améliorations apportées à la simulation de la fourniture en eau par le sol et à la réponse du blé au stress hydrique, il subsiste, même en conditions contrôlées, un écart entre l'observation et le modèle. Pour partie, il convient de préciser que les conditions d'écoulement de l'eau dans les lysimètres ne sont pas celles du champ et qu'il existe des circuits préférentiels (parois, tube neutronique) qui pourraient expliquer les écarts observés en matière de volume drainé (résultats non présentés).

L'effet du stress hydrique sur le développement du système racinaire n'est pas considéré directement par Epicphase, ce qui peut se traduire par un excès de consommation simulée lié à la simulation d'une colonisation du profil trop importante ou à une mauvaise estimation de MST. Par ailleurs, les mécanismes d'adaptation à la sécheresse ne sont pas considérés : il peut s'agir d'ajustement osmotique ou de mise à contribution des réserves de la tige lors du remplissage, processus qui permettent d'augmenter l'efficience pour la production de biomasse ou de grain (Turner, 1986). S'inspirant de l'approche semi-empirique menée sur tournesol (Texier, 1992) et inscrite dans les fonctions du modèle, il serait possible de traduire les proces- 
sus d'adaptation à la sécheresse par divers paramètres: i) une modification du besoin hydrique liée à l'ajustement osmotique ; ii) un effet différentiel sur l'indice de récolte selon l'intensité du stress, permettant une mise en réserve et une translocation préférentielle d'assimilats depuis la tige.

La fonction linéaire décrivant la progression des racines n'est pas encore satisfaisante, malgré les ajustements pratiqués : une fonction sigmoïdale ralentissant la progression des racines avant le tallage et après l'anthèse serait plus conforme aux observations (Massé et al, 1988).

Un autre point à parfaire concerne le fonctionnement hivernal de la culture et la simulation précoce des cinétiques d'indice foliaire et de biomasse. Steduto et al (1995) ont montré les limites du modèle Epic pour le blé d'hiver dans un contexte méditerranéen où il n'y a pas d'arrêt de végétation en hiver. Par ailleurs, l'ajustement de la fonction de croissance n'est pas satisfaisant lors de printemps froids (Cabelguenne et al, 1990). C'est pourquoi le modèle ne simule pas correctement la biomasse et l'indice foliaire au début du printemps, ce qui limite son utilisation pour la prévision de la biomasse aérienne début montaison.

Le découpage du cycle en phases de sensibilité variable aux contraintes hydrique et azotée a été introduit dans Epicphase sur la seule base des sommes de température. En cultures d'hiver, la simulation des durées de développement nécessite l'introduction d'effets vernalisants et photopériodiques (Brisson et Delécolle, 1991) que le modèle ignore. Cette lacune se traduit par une prévision imparfaite de la durée "semisstade épi $1 \mathrm{~cm}$ " (fig 6b). Néanmoins, l'incidence des contraintes hydriques et azotées sur l'indice de récolte est peu marquée au cours des phases 1 et 2 , ce qui tamponne les conséquences d'une erreur sur la détermination de la durée des phases. L'objectif du modèle n'est pas de prédire des durées de développement en tant que telles, d'autant plus que l'effet du stress hydrique sur la durée des phases n'est pas considéré explicitement. Dans la phase 4 , néanmoins, la simulation de la cinétique accélérée de sénescence foliaire (fig 4) conduit à un arrêt plus précoce du remplissage $(I F=0)$ et donc à une date de maturité avancée. Par ailleurs, la bonne simulation de la durée "semis-épiaison" (fig $6 \mathrm{c}$ ) suggère que l'action du déficit hydrique sur la durée des phases n'est pas très sensible avant l'épiaison du blé.

\section{Les paramètres d'entrée du modèle sont peu mesurables ou dérivés d'un petit nombre de références ou d'expériences}

Le rapport énergie/biomasse (RUE) est un paramètre auquel le modèle est très sensible (fig 5). Cette valeur est strictement dérivée de la littérature et varie beaucoup selon les auteurs (Russell et Wilson, 1994). Ruget et al (1991) signalent plusieurs causes de discordance possibles entre valeurs de RUE retenues par les modèles mécanistes et réalité du champ : i) les références portent souvent sur les seules parties aériennes ce qui sous-estime les valeurs de RUE données par la littérature ; ii) la valeur de RUE ne peut être considérée comme constante avec le développement foliaire, la variation de composition biochimique de la plante et l'intervention croissante de la respiration d'entretien; iii) en conditions sèches et chaudes, la valeur de RUE pourrait être affectée par l'augmentation des taux de respiration. Seuls des stress hydriques et thermiques marqués paraissent affecter la valeur de RUE pour le blé d'hiver (Gallagher et Biscoe, 1978 ; Green, 1984 ; Howell et Musick, 1985 ; Steinmetz et al, 1990). La partition entre biomasse aérienne et racinaire, basée sur une évolution simple du rapport correspondant au cours du cycle, indépendamment de l'alimentation hydrique, est une cause d'erreur dans l'estimation de MST (Barraclough, 1984). Notons également que des pertes de biomasse d'environ 10 $\%$ ont été observées entre la fin de remplissage du grain et la récolte, liées à la respiration de la tige et de l'épi ou aux pertes de tissus sénescents (Russell et Wilson, 1994), processus ignorés par le modèle. Enfin, les variations du coefficient d'extinction ( $k$ ) au cours du cycle (Asrar et Kanemasu, 1985) ajoutent une cause d'erreur supplémentaire, quoique mineure. Une valeur de RUE de 2,8 à $3,0 \mathrm{~g} \cdot \mathrm{MJ}^{-1}$ (tenant compte des racines) paraît convenable en conditions hydriques non limitantes (Gallagher et Biscoe, 1978), alors qu'une valeur de 2,3 à $2,5 \mathrm{~g} \cdot \mathrm{MJ}^{-1}$ permet de mieux rendre compte des situations sèches (Steiner et al, 1987). Au regard de la littérature (Green, 1984 ; Asrar et Kanemasu, 1985), il paraît concevable de proposer pour le blé une évolution de RUE par phase, en conditions potentielles de croissance : $70 \%$ de RUE max (phase 1), RUE max (phase 2), $85 \%$ de RUE max (phase 3), décroissance régulière en phase 4 (avec la sénescence physiologique). La structure d'Epicphase permet cette adaptation. Dans un second temps, les effets des stress thermique et hydrique sur RUE pourraient être introduits 
selon le schéma proposé par Russell et Wilson (1994) : variation linéaire en deçà et au-delà de la plage de température optimale $\left(10-25^{\circ} \mathrm{C}\right)$, réduction de RUE lorsque la réserve facilement utilisable est consommée.

La plupart des tests effectués sur le modèle Epic et concernant le blé d'hiver se sont limités au rendement voire à la biomasse totale (Steiner et al, 1987 ; Williams et al, 1989 ; Cabelguenne et al, 1990 ; Favis-Mortlock et al, 1991 ; Moulin et Beckie, 1993). Dans ce travail, nous avons tenté d'explorer plus finement le fonctionnement du modèle sous contrainte hydrique. La comparaison directe avec la version originale d'Epic souligne le progrès accompli (tableau III). La méthode semi-empirique utilisée, séparant les effets du déficit hydrique sur la biomasse totale d'une part, sur l'indice de récolte d'autre part, nous a permis de corriger les défauts du modèle sans trop en complexifier la structure initiale. La bonne simulation de la dynamique des stocks hydriques du sol et de la réponse du blé au déficit par phase apparaissent compatibles avec l'utilisation du modèle Epicphase pour la définition de stratégies de conduite de la culture en conditions plus ou moins limitantes en eau.

\section{RÉFÉRENCES}

Aase JK (1978) Relationship between leaf area and dry matter in winter wheat. Agron J 70, 563-565

Asrar G, Kanemasu ET (1985) Seasonal distribution of water use and photosynthetic efficiencies of winter wheat. Conf Int "Les besoins en eau des cultures», Paris, 277-283

Baker DN, Whisler FD, Parton WJ, Klepper EL, Cole CV, Willis WO, Smika DE, Black AL, Bauer A (1985) The development of WINTER WHEAT a physiological process model. USDA-ARS Wheat Yield Project, ARS-38, 176-187

Barraclough D (1984) The growth and activity of winter wheat roots in the field: root growth of high-yielding crops in relation to shoot growth. $J$ Agric Sci Camb $103,439-442$

Bouthier A, Bonnefoy M (1993) Céréales : une pratique à adapter selon les sols et les espèces. In : Gérer l'irrigation en grandes cultures, ITCF, 6-14

Brisson N, Delécolle R (1991) Développement et modèles de simulation de culture. agronomie 12 , 253-263

Brisson N, Delécolle R (1993) Utilisation des modèles mécanistes de culture comme outils de raisonnement de la composante génétique de la résistance à la sécheresse. In: Tolérance à la sécheresse des céréales en zone méditerranéenne. Diversité génétique et amélioration variétale, Montpellier (France),
15-17 décembre 1992, Éd INRA, Paris, Les Colloques $n^{\circ} 64,117-125$

Cabelguenne M, Jones CA, Marty Jr, Quinones H (1988) Contribution à l'étude des rotations culturales : tentative d'utilisation d'un modèle. agronomie $8,549-556$

Cabelguenne $M$, Jones CA, Marty JR, Dyke P, Williams PT (1990) Calibration and validation of EPIC for crop rotations in southern France. Agric Syst 33, 153-171

Cabelguenne M, Debaeke P, Puech J (1994) Simulation de stratégies et de tactiques d'irrigation en conditions de ressources en eau limitées. 17th Europ Reg Conf Irrig Drain ICID, Varna (Bulgarie), 16-22 mai 1994, 39-48

Castillon P, Chabanel Y, Massé J, Lescot MP (1986a) Produire de la paille en culture intensive des céréales ; influence du milieu et des techniques culturales. Forum "Céréales à paille", Midi-Pyrénées, Persp Agric, $n^{\circ}$ Hors Série, juin 1986, 70-76

Castillon P, Duboin G, Noblot JM (1986b) Raisonnement de la fertilisation azotée des céréales dans le SudOuest. Forum "Céréales à paille», Midi-Pyrénées, Persp Agric, $\mathrm{n}^{\circ}$ Hors Série, juin 1986, 50-54

Dagneaud JP, Tranchefort J (1985) Prévision des rendements du blé tendre d'hiver à l'aide d'un modèle climatique. CR Acad Agric Fr 71, 911-916

Debaeke P, Hilaire A (1991) Étude expérimentale de systèmes de culture extensifs et modèles de simulation. Séminaire "L'extensification, une forme de modernisation", Dijon, $23-24$ oct 1990, CIFAR Éd, Paris, 136-146

Debaeke P, Puech J, Casals ML (1996) Élaboration du rendement du blé d'hiver en conditions de déficit hydrique. I. Étude en lysimètres. agronomie 16, 3-23

Donald CM, Hamblin J (1976) The biological yield and harvest index of cereals as agronomic and plant breeding criteria. Adv Agron 28, 361-405

Duboin G (1986) Adaptation des variétés aux petites régions et à la date de semis. Méthode de choix d'un type de variété. Forum "Céréales à paille», Midi-Pyrénées, Persp Agric, HS juin 1986, 38-44

Farshi AA, Feyen J, Belmans C, de Wijngaert K (1987) Modelling of yield of winter wheat as a function of soil water availability. Agric Water Management 12 , 323-339

Favis-Mortlock DT, Evans R, Boardman J, Harris TM (1991) Climate change, winter wheat yield and soil erosion on the English South Downs. Agric Syst 37, 415-433

Fischer RA (1985) The role of crop simulation models in wheat agronomy. In: Wheat Growth and Modelling (W Day, RK Atkin, eds), Series A : Life Sciences 86, 237-251, Plenum Press, New York

Fuchs M, Asrar G, Kanemasu ET (1984) Leaf area estimates from measurements of photosynthetically active radiation in wheat canopies. Agric For Meteoro/ 32, 13-22

Gallagher JN, Biscoe PV (1978) Radiation absorption, growth and yield of cereals. J Agric Sci Camb 91, 47-60 
Garcia RG, Kanemasu ET, Blad BL, Bauer A, Hatfield JL, Major DJ, Reginato RJ, Hubbard KJ (1988) Interception and use efficiency of light in winter wheat under different nitrogen regimes. Agric For Meteorol 44, 175-186

Gate P, Massé J (1990) Forecasting winter wheat yield in the field future prospects. Proc ESA First Congress, Paris, 2, 12

Gosse G, Varlet-Grancher C, Bonhomme R, Chartier M, Allirand JM, Lemaire G (1986) Production maximale de matière sèche et rayonnement solaire intercepté par un couvert végétal. agronomie 6, 47-56

Green CF (1984) Discriminants of productivity in small grain cereals: a review. J Natn Inst Agric Bot 16, 453-463

Green CF (1989) Genotypic differences in the growth of Triticum aestivum in relation to absorbed solar radiation. Field Crops Res 19, 285-295

Gregory PJ, McGowan M, Biscoe PV, Hunter B (1978) Water relations of winter wheat. I. Growth of the root system. J Agric Sci Camb 91, 91-102

Hansen S, Jensen HE, Nielsen NE, Svendsen H (1991) Simulation of nitrogen dynamics and biomass production in winter wheat using the Danish simulation model DAISY. Fertilizer Res 27, 245-259

Howell TA, Musick JT (1985) Relationships of dry matter production of fields crops to water consumption. Conf Int "Les besoins en eau des cultures", Paris, 247-269

Jones CA (1983) A survey of the variability in tissue nitrogen and phosphorus concentrations in maize and grain sorghun. Field Crops Res 6, 133-147

Keulen van H, Seligman NG (1987) Simulation of growth, water use and nitrogen nutrition of spring wheat. Simulation Monographs, Pudoc, Wageningen, Pays-Bas

Kiniry JR, Williams JR, Gassman PW, Debaeke P (1992) A general, process-oriented model for two competing plant species. Trans ASAE 35, 801-810

Littleboy M, Silburn DM, Freebairn DM, Woodruff DR, Hammer GL (1989) PERFECT, a computer simulation model of productivity erosion runoff functions to evaluate conservation techniques, Queensland Dept of Primary Industries, Brisbane, $119 \mathrm{p}$

Maas SJ, Arkin GF (1980) TAMWM, a wheat growth and development simulation model. Program and model doc $n^{\circ}$ 80-3, TAES, Blackland Research Center, Temple, États-Unis

Maertens C, Cabelguenne M (1974) Intensité et limite de dessèchement du sol en relation avec l'enracinement de quelques espèces cultivées. CR Acad SC Paris 279, 2039-2042

Maertens C, Clauzel Y (1988) Résultats obtenus par endoscopie. In : Les racines, résultats acquis en plein champ, Persp Agric, 128, 55-57

Maertens C, Marty JR (1972) Contrôle de l'utilisation et de l'efficience de l'eau chez diverses cultures irriguées ou non, au moyen d'humidimètres à neutrons. "Isotopes and radiation in soil-plant relationships including forestry", IAEA, Vienne, 621-630
Massé J, Colnenne C, Tardieu F, Crosson P (1988) Système racinaire du blé et état structural du sol. In: Les racines, résultats acquis en plein champ, Persp Agric 128, 31-37

Monteith $J L$ (1977) Climate and efficiency of crop production in Britain. Phil Trans $R$ Soc Lond B 281, 277-294

Moulin AP, Beckie HJ (1993) Evaluation of the CERES and EPIC models for predicting spring wheat grain yield over time. Can J Plant Sci 73, 713-719

O'Leary GJ, Connor DJ, White DH (1985) A simulation model of the development, growth and yield of the wheat crop. Agric Syst 17, 1-26

Paillard C, Massé J, Robert D, Crosson P (1992) Blé d'hiver : modélisation de la progression racinaire. Persp Agric 175, 112-115

Porter JR (1993) AFRCWHEAT2: a model of the growth and development of wheat incorporating responses to water and nitrogen. Eur J Agron 2, 69-82

Porter JR, Jamieson PD, Wilson DR (1995) Comparison of the wheat simulation models AFRCWHEAT2, CERES-Wheat and SWHEAT for non-limiting conditions of crop growth. Field Crops Res 33, 131-157

Puech J (1972) Étude expérimentale de la disponibilité de l'eau pour les végétaux sur différents types de sols. Thèse, univ P-Sabatier, Toulouse, $161 p$

Quinones Pedroza HE (1989) Simulation des contraintes hydriques sur la croissance et le rendement du tournesol et du maïs. Thèse, INP, Toulouse, $172 p$

Rasmussen VP, Hanks RJ (1978) Spring wheat yield model for limited moisture conditions. Agron $J 70$, 940-944

Ritchie JT (1972) Model for predicting evapotranspiration from a row crop with incomplete cover. Water Resour Res 8, 1204-1213

Ritchie JT, Otter S (1985) Description and performance of CERES-Wheat yield model: a user-oriented wheat yield model. USDA-ARS Wheat Yield Project, 159-175

Ruget F, Bonhomme R, Varlet-Grancher C (1991) Analyse de la fonction de photosynthèse dans CERES-Maize. In: Physiology et production du maïs (D Picard, ed), INRA Éditions, 445-453

Russell G, Wilson GW (1994) An agro-pedo-climatological knowledge-base of wheat in Europe. EC Publication, Luxembourg, $158 \mathrm{p}$

Singels A (1992) Evaluating wheat planting strategies using a growth model. Agric Syst 38, 175-184

Stapper M, Harris HC (1989) Assessing the productivity of wheat genotypes in a Mediterranean climate, using a crop-simulation model. Field Crops Res 20, 129-152

Steduto P, Pocuca V, Caliandro A, Debaeke P (1995) Test on the crop-growth simulation submodel of EPIC. Case of wheat grown under Mediterranean climate and variable soil-water regimes. Eur $J$ Agron 4, 335-345 
Steiner JL, Williams JR, Jones OR (1987) Evaluation of EPIC stimulation model using a dryland wheatsorghum-fallow crop rotation. Agron J 79, $732-738$

Steinmetz S, Guerif M, Delécolle R, Baret F (1990) Spectral estimates of the absorbed photosynthetically active radiation and light use efficiency of a winter wheat crop. Int J Remote Sensing 11, 1797-1808

Stockle CO, Campbell GS (1989) Simulation of crop response to water and nitrogen: an exemple using spring wheat. Trans ASAE 32, 66-74

Stockle CO, Martin SA, Campbell GS (1994) CropSyst, a cropping systems simulation model water and nitrogen budgets and crop yield. Agric Syst 46, 335-359

Szeicz G (1974) Solar radiation for plant growth. $J$ Appl Ecol 11, 617-636

Texier $V$ (1992) Croissance et production du tournesol dans diverses conditions de milieu : étude expérimentale et modélisation. Thèse, univ P-Sabatier, Toulouse, $125 p+$ annexes

Turner NC (1986) Crop water deficits a decade of progress. Adv Agron 39, 1-51

Weir AH, Bragg PL, Porter JR, Rayner JH (1984) A winter wheat crop simulation model without water or nutrient limitations. J Agric Sci Camb 102, 371-382

Whisler FD, Acock B, Baker DN, Fye RE, Hodges HF, Lambert JR, Lemmon HE, McKinion JM, Reddy VR (1986) Crop simulation models in agronomic systems. Adv Agron 40, 141-208

Williams JR, Jones CA, Dyke PT (1984) A modeling approach to determining the relationship between erosion and soil productivity. Trans ASAE 27, 129144

Williams JR, Jones CA, Kiniry JR, Spanel DA (1989) The EPIC crop growth model. Trans ASAE 32, 497511

Willmot CJ (1982) Some comments on the evaluation of model performance. Bull Am Meteorol Soc 63, 1309-1313

\section{ANNEXE 1. MODĖLE DE FONCTIONNEMENT DE LA CULTURE EN L'ABSENCE DE STRESS}

\section{Croissance sans facteurs limitants autres que le rayonnement incident}

(1) $\mathrm{MST}_{j}=\mathrm{MST}_{j-1}+\Delta M S T_{\text {pot }}$

avec $\triangle M S T_{\text {pot }}=0.001 * R U E_{j} * I P A R_{j}^{*}(1+$ $\triangle \mathrm{DJOUR})^{3}$

et $\operatorname{IPAR}_{j}=a^{*} \mathrm{Rg}_{j}{ }^{*}\left[1-\exp \left(-\mathrm{k}_{j}{ }^{*} \mathrm{IF}_{j}\right)\right]$

$\Delta \mathrm{X}=$ variation journalière de la variable $X$ entre $j$-1 et $j$

MST = biomasse totale (racines comprises), en t.ha ${ }^{-1}$
$\mathrm{RUE}_{j}=$ efficience de conversion du PAR intercepté en biomasse (jour $l$ ) en $\mathrm{kg}$ ha-1 $\mathrm{MJ}^{-1} \mathrm{~m}^{2}$ (levée-début sénescence)

$\mathrm{IPAR}_{j}=$ rayonnement photosynthétiquement actif intercepté par le couvert en $\mathrm{MJ} . \mathrm{m}^{-2}$

DJOUR $_{j}=$ durée du jour $j(\mathrm{~h})$

$a=$ paramètre de conversion de Rg en PAR (incident), fixé à 0,5 par Szeicz (1974)

$\mathrm{Rg}_{j}=$ rayonnement global incident en $\mathrm{MJ} . \mathrm{m}^{-2}$ (mesuré)

$\mathrm{k}_{j}=$ coefficient d'extinction de la lumière (jour $j$ )

$\mathrm{IF}_{j}=$ indice foliaire (jour $j$ )

À partir de la sénescence, $\mathrm{RUE}_{j}$ est remplacé par RUEsen : $^{2}$

(2) $R U E s e n_{j}=$ RUE $_{j}{ }^{*} 10\left[\right.$ dRUE $\left.{ }^{*} \log \left(1.001-\mathrm{RST}_{j} / 1-\mathrm{RST}_{m x}\right)\right]$

dRUE = paramètre de déclin de RUE au cours de la sénescence foliaire

$\mathrm{RST}_{j}=$ rapport de la somme des températures de l'air (base $\mathrm{T}_{b}$ ) au jour $j$ à la somme des températures à maturité $\left(\mathrm{RST}_{m x}<\mathrm{RST}_{j}<1\right)$

$\mathrm{RST}_{m x}=$ valeur de RST correspondant au début de la sénescence foliaire

\section{Cinétique d'indice foliaire}

\section{En phase d'établissement}

(3) $\mathrm{IFpot}_{j}=\mathrm{IF}_{m x}{ }^{*} \mathrm{RST}_{j} /\left[\mathrm{RST}_{j}+\exp \left(\mathrm{a} 1-\mathrm{a} 2{ }^{*} \mathrm{RST}_{j}\right)\right]$

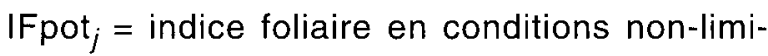
tantes (jour $j$ )

$\mathrm{IF}_{m x}=$ indice foliaire maximal accessible pour la densité de peuplement réalisée

a1, a2 = paramètres d'ajustement

\section{En phase de sénescence RST $\left._{j}>\mathrm{RST}_{m \boldsymbol{x}}\right)$}

(4) $\mathrm{IFpot}_{j}=\mathrm{IF}_{m x}{ }^{*} 10\left[\mathrm{dIF}{ }^{*} \log \left(1.001-R S \mathrm{~T}_{j} / 1-\mathrm{RST}_{m x}\right)\right]$

$\mathrm{dIF}=$ paramètre de déclin de l'indice foliaire

\section{Cinétique de progression de l'enracinement}

$$
\text { (5) } P E_{j}=V^{*} P M A X * R S T_{j}
$$

$\mathrm{PE}_{j}=$ profondeur d'enracinement au jour $j(\mathrm{~m})$

$V=$ paramètre de progression du front racinaire $\left(\mathrm{m} \cdot \mathrm{m}^{-1}\right)$

PMAX = profondeur maximale d'enracinement (m) 
Répartition de la biomasse entre organes

(6) $M S R_{j}=M S T_{j}{ }^{*}\left(\mathrm{~b} 1-\mathrm{b} 2{ }^{*} R S T_{j}\right)$

(7) $M S G_{j}=\mathrm{IR}_{0}{ }^{*} M S A_{j}$ (à maturité)

$\mathrm{MSR}_{j}=$ matière sèche racinaire (t.ha- $\left.{ }^{-1}\right)$

$M S G_{j}=$ matière sèche du grain (t.ha- $\left.{ }^{-1}\right)$

$\mathrm{MSA}_{j}=$ matière sèche aérienne (t.ha ${ }^{-1}$ )

$I_{0}=$ indice de récolte en l'absence de stress hydrique et azoté

b1, b2 = paramètres d'ajustement

\section{Teneur azotée optimale de la plante}

(8) $\mathrm{C}_{\mathrm{NBj}}=\mathrm{c} 1+\mathrm{c} 2{ }^{*} \exp \left(-\mathrm{c} 3{ }^{*} R S T_{j}\right)$

$C_{N B j}=$ teneur azotée optimale de la biomasse totale au jour $j$

c1, c2, c3 = paramètres d'ajustement

\section{Prélèvement d'eau sans facteurs limitants autres que la demande climatique et la profondeur d'enracinement}

(9) $\mathrm{FE}_{p 1, j}=\left[\mathrm{ETM}_{j} /(1-\exp (-\alpha))\right]^{\text {* }}$

$$
\sum_{h=1}^{10}\left[1-\exp \left(-\alpha^{*} z_{h} / R Z\right)\right]
$$

$\mathrm{FE}_{p 1, j}=$ fourniture en eau potentielle journalière sur la profondeur de sol $z_{h}$, limitée par la profondeur d'enracinement $R Z(\mathrm{~mm})$

$\mathrm{ETM}_{j}=$ évapotranspiration maximale du jour (besoin hydrique), en $\mathrm{mm}$

$\alpha=$ paramètre de distribution de l'extraction d'eau (ou de forme d'enracinement efficace)

$h=$ horizon de sol (de 1, le plus superficiel à 10, le plus profond)

$$
\begin{gathered}
\text { (10) } \mathrm{ETM}_{j}=\mathrm{ETP}_{j}^{*}\left(\mathrm{IF}_{j} / 3\right) \\
\text { si } 0 \leq \mathrm{IF}_{j} \leq 3 \mathrm{ETM}_{j}=\mathrm{ETP}_{j} \text { si IF } \\
\text { (11) } \mathrm{FE}_{p 2, j}=3 \\
{\left[1-\exp \left(-\alpha E_{p 1, j}-\sum_{\mathrm{h}=1}^{10}\left[(1-\mathrm{p})^{*}\right.\right.\right.} \\
\left.[1-1 / \mathrm{RZ})]-\mathrm{p}^{*} \sum_{\mathrm{k}=1}^{\mathrm{h}-1} \mathrm{FE}_{k}\right]
\end{gathered}
$$

$\mathrm{FE}_{p 2, j}=$ fourniture en eau potentielle journalière sur la profondeur de sol $z_{h}$, limitée par la profondeur d'enracinement $R Z$ et tenant compte de la participation des autres horizons de sol $(\mathrm{mm})$ $p=$ paramètre de compensation de l'alimentation hydrique par les autres horizons du sol (0-1) $\mathrm{FE}_{k}=$ fourniture en eau réelle par les horizons supérieurs à $h(\mathrm{~mm})$

\section{ANNEXE II. RÉPONSE DE LA CULTURE AUX STRESS HYDRIQUES ET AZOTÉS}

\section{Réponse de la biomasse totale au stress hydrique}

$$
\begin{aligned}
& \text { (12) } \mathrm{ETR}_{j}=\sum_{h=1}^{10} \mathrm{FE}_{p 2, h}{ }^{*} \exp \left(5^{*} \mathrm{t}^{*}\right. \\
& \left.\left(\mathrm{H}_{h, j}-\mathrm{HPF}_{h} / \mathrm{HCC}_{h}-\mathrm{HPF}_{h}\right)-5\right)
\end{aligned}
$$

avec: $\mathrm{H}_{h, j}<\left(\left(\mathrm{HCC}_{h}-\mathrm{HPF}_{h}\right) / \mathrm{t}\right)+\mathrm{HPF}_{h}$

$\mathrm{ETR}_{j}=$ évapotranspiration réelle $(\mathrm{mm})$

$\mathrm{H}_{h, j}=$ humidité volumique du sol (horizon $h$, jour J), en $\%$

$\mathrm{HCC}_{h}=$ humidité à la capacité au champ (\%)

$\mathrm{HPF}_{h}=$ humidité au point de flétrissement (\%)

$t=$ paramètre de cessibilité de l'eau

(13) $\Delta \mathrm{MST}=\Delta \mathrm{MST}_{\text {pot }}{ }^{*}\left(\mathrm{ETR}_{j} / \mathrm{ETM}_{j}\right)$

\section{Réponse de la biomasse totale au stress azoté}

(14) $\mathrm{SN}_{j}=1-\left[\mathrm{sNj} /\left(\mathrm{sN}_{j}+\exp \left(\mathrm{d} 1-\mathrm{d} 2{ }^{*} \mathrm{SN}_{j}\right)\right)\right]$

(15) $\mathrm{sN}_{j}=2$ * $\left(1-\left[\mathrm{NABS}_{j} /\left(\mathrm{cNB}_{j}{ }^{*} \mathrm{MST}_{j}\right)\right]\right.$

(16) $\Delta \mathrm{MST}=\Delta \mathrm{MST}_{\text {pot }}{ }^{*} \mathrm{SN}_{j}$

$\mathrm{SN}_{j}=$ taux de satisfaction du besoin azoté $(0,5-$ 1)

NABSj = quantité d'azote absorbée par le blé au jour $j\left(\mathrm{~kg} / \mathrm{ha}^{-1}\right)$

d1,$d 2=$ paramètres d'ajustement

\section{Réponse de l'indice foliaire aux stress hydriques et azotés}

(17) $\Delta \mathrm{IF}=\Delta \mathrm{IFpot}{ }^{*}\left[1-\exp \left(5^{*}\left(\mathrm{IF}_{j-1}-\mathrm{IF}_{m x}\right)\right)\right]{ }^{*} \mathrm{VFLIM}_{j}$ avec $\mathrm{FLIM}_{j}=\min \left(\mathrm{SN}_{j}, \mathrm{ETR}_{j} / \mathrm{ETM}_{j}\right)$

$\mathrm{FLIM}_{j}=$ facteur du limitant du jour $j$, hydrique ou azoté ( $0=$ stress maximal à $1=$ pas de stress). 